Annals of Pure and Applied Mathematics

Vol. 17, No. 2, 2018, 163-191

ISSN: 2279-087X (P), 2279-0888(online)

Published on 2 June 2018

www.researchmathsci.org

DOI: http://dx.doi.org/10.22457/apam.v17n2a3

Annals of

Pure and Applied

Mathematics

\title{
On the Field Derivatives and its Application to Fluids
}

\author{
Javier Rivera \\ 865 River Boat Circle, Orlando, FL 32828 \\ USA Copyright Registration \# TXu 2-074-440 \\ Email: caribe31415@gmail.com
}

Received 9 April 2018; accepted 31 May 2018

\begin{abstract}
This article contains the fundamental theorems and definitions of the field derivative operator. Theorem 1, Field derivative of a field function and Theorem 2, Fundamental Theorem of Calculus for the field derivative proven for functions defined within a fluid velocity field, in short, a field function. The field derivative has the same properties and rules as the material derivative and the total derivative. The field derivative is neither a covariant derivative nor a Lie derivative since it does not involve parallel transport of vectors, but field derivative is an ordinary directional derivative in phase-space-time domain ( 7 dimensions). The field derivative of the Bernoulli equation yields the addition of the fluid components multiplied by its respective Navier Stokes equations and therefore is constant throughout every point in the fluid 1-dimensional stream. Additionally, the definition and examples of the field derivative are demonstrated, which are essential to demonstrate the first integral of the Navier Stokes is the Bernoulli equation for viscous fluids through a sampling scheme. The development of the Hamilton Jacobi partial differential equation of incompressible fluids is based on the theorem of Gelfand and Fomin's book Calculus of Variations was adapted to fluid mechanics notation using the field derivative operator instead of the total derivative used in this theorem. When considering the Bernoulli formulas in this manuscript, the Eulerian coordinates were used for small volumes with a sampling scheme. By construction of the sampling scheme and the use of the induction method, the Eulerian stream control volume tended to zero, since the stream control volumes became Lagrangian fluid parcels or particles within a 1-dimensional stream. Therefore, the integral of the field derivative of the Bernoulli function is integrable and depends only on the time limits of the line integral.
\end{abstract}

Keywords: Bernoulli's principle, incompressible viscous fluids, field derivative, Hamilton-Jacobi PDE

AMS Mathematics Subject Classification (2010): 76A02

\section{Introduction}

The article entitled "A variational principle for incompressible viscous fluids" was presented at the Twenty-First Southeastern Conference on Theoretical and Applied Mechanics in Orlando, Florida [1]. The innovation of this article was to add half of the fluid's internal mechanical energy into the Lagrangian of the incompressible fluid to 


\section{Javier Rivera}

derive the Navier-Stokes equations via the calculus of variations technique using the epsilon-delta continuity definition for continuous functions. The Hamiltonian was obtained via the use of the Legendre transformation, yet the resulting Hamiltonian is no longer the total mechanical energy per unit mass; rather, it is a different thermodynamic quantity. The article's thermodynamic consideration section showed that the total mechanical energy becomes a constant, in other words, the first integral of motion, similar to Bernoulli's principle for inviscid fluids. The difficulty centered on treating the fluid's Lagrangian and Hamiltonian integrands without using the fluid's volume integral and the understanding the nature of the total derivative of the Bernoulli equation (see Appendix A and reference [6]). The aim of this manuscript is to demonstrate how Bernoulli's principle for incompressible viscous fluids can be formally obtained. Additionally, the definition and examples of the field derivative are demonstrated in Section 2. One of the examples demonstrates the first integral of the Navier Stokes is the Bernoulli equation for incompressible viscous fluids. The Einstein summation convention is used throughout this manuscript with indices $\mathrm{j}$ and $\mathrm{i}$ equal to 1, 2 and 3, unless otherwise noted. Note the capital letter $\vec{X}$, denotes Eulerian coordinates, and the small letter $\vec{x}$ denotes Lagrangian coordinates in this manuscript.

\section{Mathematical theorems for the field derivative}

"The concept of a total derivative of a function of several independent variables does not exist in mathematics" ...

"Therefore, introducing the concept of a total derivative with respect to time in a strict mathematical sense is impossible. An additional physics-based idea postulating what spatial coordinates should be fixed and how to identify them is needed." - Ivanova et al, [6] page 5

The definition of the material derivative as used in Serrin's excellent article entitled "Mathematical Principles of Classical Fluid Mechanics" [10], Batchelor's book Introduction to Fluid Dynamics [7] and definition 6, equations 46 in reference [6], is reproduced below with slight notation changes in the argument and order of the arguments, below.

$$
\frac{D F(t, \vec{X}(t))}{D t} \equiv \lim _{\Delta t \rightarrow 0} \frac{F(t+\Delta t, \vec{X}+\Delta \vec{S})-F(t, \vec{X}(t))}{\Delta t}
$$

where $\vec{X}$ is a coordinate vector, $\Delta \vec{S}$ is a displacement vector, and $\Delta t$ is a time interval it took for the displacement of coordinate $\vec{X}$. In this manuscript we will use $D / D t$ to represent material derivative, since the symbol used in [6] is a similar notation to the variational derivative notation.

Consider the following definition of a time derivative operator defined below and where the fluid velocity is defined as $u_{j}\left(t, X_{j}(t)\right)=\frac{d X_{j}(t)}{d t}$

$$
\begin{aligned}
\frac{d F(t, \vec{X}(t))}{d t} \equiv \operatorname{limit}_{\Delta t \rightarrow 0} \frac{F(t+\Delta t, \vec{X}(t+\Delta t))-F(t, \vec{X}(t))}{\Delta t} \\
=\operatorname{limit}_{\Delta t \rightarrow 0} \frac{F\left(t+\Delta t, \vec{X}(t)+\Delta t d \vec{X}(t) / d t+O\left(\Delta t^{2}\right)\right)-F(t, \vec{X}(t))}{\Delta t}
\end{aligned}
$$


On the Field Derivatives and its Application to Fluids

By Taylor's Theorem [5], we can take the Taylor series of the spatial argument, $\vec{X}(t+$ $\Delta t$ ), then perform the Taylor series of the function,

$F\left(t+\Delta t, \vec{X}(t)+\Delta t d \vec{X}(t) / d t+O\left(\Delta t^{2}\right)\right)$, about the point $\left(t, X_{j}(t)\right)$ we obtain the following approximations, where it is clear that $\Delta \vec{S}=\Delta t d \vec{X}(t) / d t+O\left(\Delta t^{2}\right)$ by comparing with the material derivative definition above

$$
\begin{aligned}
F(t+\Delta t, \vec{X}(t)+ & \left.\Delta t d \vec{X}(t) / d t+O\left(\Delta t^{2}\right)\right) \\
& =F(t, \vec{X}(t))+\frac{\partial F}{\partial t}(t+\Delta t-t)+\frac{\partial F}{\partial X_{j}}\left(X_{j}(t)+\frac{d X_{j}(t)}{d t} \Delta t-X_{j}(t)\right)+O\left(\Delta t^{2}\right)
\end{aligned}
$$

Simplifying, keeping only terms lower than or equal to second degree in $\Delta t$, and using the definition of the fluid velocity, $u_{j}\left(t, X_{j}(t)\right)=\frac{d X_{j}(t)}{d t}$

$$
\begin{aligned}
F(t+\Delta t, \vec{X}(t)+ & \left.\Delta t d \vec{X}(t) / d t+O\left(\Delta t^{2}\right)\right)-F(t, \vec{X}(t)) \\
& =\left(\frac{\partial F}{\partial t}+u_{j}\left(t, X_{j}(t)\right) \frac{\partial F}{\partial X_{j}}\right) \Delta t+O\left(\Delta t^{2}\right)
\end{aligned}
$$

Substitute into the limit above

$$
\begin{gathered}
\frac{d F(t, \vec{X}(t))}{d t}=\operatorname{limit}_{\Delta t \rightarrow 0} \frac{F\left(t+\Delta t, \vec{X}(t)+\Delta t d \vec{X}(t) / d t+O\left(\Delta t^{2}\right)\right)-F(t, \vec{X}(t))}{\Delta t} \\
\frac{d F(t, \vec{X}(t))}{d t}=\left(\frac{\partial F}{\partial t}+u_{j}\left(t, X_{j}(t)\right) \frac{\partial F}{\partial X_{j}}\right)+\text { limit }_{\Delta t \rightarrow 0} O(\Delta t)=\left(\frac{\partial F}{\partial t}+u_{j}\left(t, X_{j}(t)\right) \frac{\partial F}{\partial X_{j}}\right) \\
=\frac{D F(t, \vec{X}(t))}{D t}
\end{gathered}
$$

As an example, consider the scalar function to be the kinetic energy per unit mass of the fluid.

$$
\frac{1}{2} \frac{d u_{i}^{2}}{d t}=u_{i}\left(\frac{\partial u_{i}}{\partial t}+u_{j}\left(t, X_{j}(t)\right) \frac{\partial u_{i}}{\partial X_{j}}\right)
$$

There seems to be more to this calculation that merits further study, especially since reference [6] stated the "total derivative" does not exist as a mathematical concept, we agree with this statement since the total derivative uses a single Taylor series approximation. Thus, call this derivative a total field derivative or field derivative, the latter is the term used in this manuscript. What's missing as Ivanova et al points out is to provide the additional physics-based idea of the field derivative operator defined from a slight modification of the above concept, which we will call the field derivative postulated on the works of Ivanova, et al [6], Serrin [10], Batchelor [7], and Levi-Cevita [8] and formalizing the field derivative with a single Taylor series limit technique by including the changes of the fluid field velocity into the limit itself. The field derivative is practically mathematically defined except for the important physically based restrictions as Ivanova et al mentions in [6], which are necessary in order to avoid confusion. By introducing an additional variable, the fluid field velocity, $u_{i}$, into the argument list of the function or formula we can formulate this intuitive differentiation process of the field derivative. Although capital letter is used for coordinates, they need not to be Eulerian coordinates, these definitions applies also to Lagrangian coordinates too. 


\section{Javier Rivera}

The limit definition and functional arguments were modified to include the fluid velocity field, rearranged, and reinterpreting the differentials as defined in Levi-Cevita's book [8] "The Absolute Differential Calculus", section Systems of Total Differential Equations. Unfortunately, Levi-Cevita did not provide the definition of the differentiation limit in his book. Additionally, Levi-Cevita did not provide the added description of restrictions on the differentiation) to make clear the differentiation intent, which can be confusing without it, this type of description is common use in Physics, especially fluid's thermodynamics (see Serrin [10]). Typically, but not always, in fluid mechanics if a function has the fluid velocity field on the right side of the equal sign it typically does not include the fluid velocity field as part of functional arguments. Following the intuitive approach above, we introduce of an additional variable, the fluid velocity field, $u_{i}$, into the argument list of a function $F(t, \vec{X}(t))$, or now a field function $F(t, \vec{X}(t), \vec{u}(t, \vec{X}(t)))$, with the order of arguments follows Gelfand \& Fomin's book Calculus of Variations [11] style. The introduction of the fluid velocity field in the argument of the function provides a way to introduce the changes of the fluid's velocity field at the same moment the function changes in a combined phase space-time tangent space, thus yields a Calculus of Variation's style limit concept in function phase-space-time domain. Note that it differs from the usual calculus of variations limit in that there is no functional, and the independent variable is also varied, therefore it is not a variational derivative. The field derivative of a field function $F(t, \vec{X}(t), \vec{u}(t, \vec{X}(t)))$ is given by the theorem below.

\section{Theorem 1. Field derivative of a field function.}

If the field derivative of a field function $F(t, \vec{X}(t), \vec{u}(t, \vec{X}(t)))$ on a continues curve (or 1dimensional stream) of a field $\vec{u}(t, \vec{X}(t))$ is defined by the limit below,

$$
\begin{aligned}
& \frac{d}{d t} F(t, \vec{X}(t), \vec{u}(t, \vec{X}(t))) \\
& \quad \equiv \lim _{\Delta t \rightarrow 0} \frac{F(t+\Delta t, \vec{X}(t+\Delta t), \vec{u}(t+\Delta t, \vec{X}(t+\Delta t)))-F(t, \vec{X}(t), \vec{u}(t, \vec{X}(t)))}{\Delta t}
\end{aligned}
$$

Then the limit of the field derivative of the field function, $F(t, \vec{X}(t), \vec{u}(t, \vec{X}))$, results in the tangent plane of phase-space-time domain is given by the following equation

$$
\begin{aligned}
\frac{d}{d t} F(t, \vec{X}(t), \vec{u}(t, \vec{X}(t))) & \\
= & {\left[\frac{\partial \mathrm{F}(t, \vec{X}(t), \vec{u}(t, \vec{X}(t)))}{\partial \mathrm{t}}\right]_{\text {fixed } \vec{X} \& \vec{u}} } \\
& +u_{j}(t, \vec{X}(t))\left[\frac{\partial F(t, \vec{X}(t), \vec{u}(t, \vec{X}(t)))}{\partial X_{j}}\right]_{\text {fixed } t} \\
& +\frac{\partial u_{i}(t, \vec{X}(t))}{\partial t}\left[\frac{\partial F(t, \vec{X}(t), \vec{u}(t, \vec{X}(t)))}{\partial u_{i}}\right]_{\text {fixed } t}
\end{aligned}
$$

The field function can be a scalar field, $F$, a vector field, $F_{\mathrm{k}}$, or tensor density field, $F_{\text {ik }}$. The calculus time derivative operator symbol is used for simplicity and since the field derivative becomes calculus's total derivative of a function (i.e. using chain rule) if there 
On the Field Derivatives and its Application to Fluids

are no field components of $\vec{u}(t, \vec{X}(t))$ on both side of the equal sign in the definition of the function being differentiated. Thus, the field derivative has the same properties or rules as calculus total derivative.

Proof of Theorem 1. Field derivative of a field function.

The mathematical definition of the field derivative is given as

$$
\begin{aligned}
\frac{d}{d t} F(t, \vec{X}(t), \vec{u}(t, \vec{X}(t))) & \\
& \equiv \lim _{\Delta t \rightarrow 0} \frac{F(t+\Delta t, \vec{X}(t+\Delta t), \vec{u}(t+\Delta t, \vec{X}(t+\Delta t)))-F(t, \vec{X}(t), \vec{u}(t, \vec{X}(t)))}{\Delta t}
\end{aligned}
$$

Let $t=t(\tau)$ a $\tau$ parameter in the real line, then relabeled the following variables, for

$$
j=0, \quad Y_{0}(\tau)=t(\tau),
$$

and for

$$
j \text { within } 3 \geq j \geq 1, Y_{j}(\tau)=X_{j}(t(\tau)) \text {, and for } i \text { within } 6 \geq i \geq 4, Y_{i}(\tau)=u_{i-3}\left(t(\tau), Y_{j}(\tau)\right)
$$

Then the functional difference maybe written as for $\mathrm{k}=0$ to 6 , then the numerator can be written as

$$
\begin{gathered}
F(t+\Delta t, \vec{X}(t+\Delta t), \vec{u}(t+\Delta t, \vec{X}(t+\Delta t)))-F(t, \vec{X}(t), \vec{u}(t, \vec{X}(t))) \\
=F\left(Y_{k}(\tau+\Delta \tau)\right)-F\left(Y_{k}(\tau)\right)
\end{gathered}
$$

By Taylors Theorem for multi-dimensional spaces, let the Taylor series about point $\left(Y_{0}(\tau), Y_{1}(\tau), \ldots, Y_{6}(\tau)\right)$ be given by

$F\left(Y_{k}(\tau+\Delta \tau)\right)=F\left(Y_{k}(\tau)\right)+\sum_{k=0}^{6} \frac{\partial F}{\partial Y_{k}}\left(Y_{k}(\tau+\Delta \tau)-Y_{k}(\tau)\right)+O\left(\left(\left(Y_{k}(\tau+\Delta \tau)-Y_{k}(\tau)\right)^{2}\right)\right.$

Now, the limit is simply an ordinary time derivative limit about point, $Y_{k}(\tau)$, in $\mathrm{Y}$ space.

$$
\frac{d F\left(Y_{k}(\tau)\right)}{d \tau} \equiv \lim _{\Delta \tau \rightarrow 0} \frac{F\left(Y_{k}(\tau+\Delta \tau)\right)-F\left(Y_{k}(\tau)\right)}{\Delta \tau}
$$

Substituting the expansion of the Taylor series of $F\left(Y_{k}(\tau+\Delta \tau)\right)$ results in

$$
\begin{aligned}
& \frac{d F\left(Y_{k}(\tau)\right)}{d \tau}=\sum_{k=0}^{6} \frac{\partial F}{\partial Y_{j}} \lim _{\Delta \tau \rightarrow 0} \frac{\left(Y_{k}(\tau+\Delta \tau)-Y_{k}(\tau)\right)}{\Delta \tau}+O\left(\operatorname { l i m } _ { \Delta \tau \rightarrow 0 } \left(Y_{k}(\tau+\Delta \tau)\right.\right. \\
& \left.\left.\quad-Y_{k}(\tau)\right) \frac{\left(Y_{k}(\tau+\Delta \tau)-Y_{k}(\tau)\right)}{\Delta \tau}\right)
\end{aligned}
$$

Since $\lim _{\Delta \tau \rightarrow 0}\left(Y_{k}(\tau+\Delta \tau)-Y_{k}(\tau)\right)=0$, and $\frac{\partial Y_{k}}{\partial \tau}=\lim _{\Delta \tau \rightarrow 0} \frac{\left(Y_{k}(\tau+\Delta \tau)-Y_{k}(\tau)\right)}{\Delta \tau}$ due to the fact some of the functions may depend on both time, and coordinates, not shown.

$$
\frac{d F\left(Y_{i}(\tau)\right)}{d \tau}=\sum_{k=0}^{6} \frac{\partial F}{\partial Y_{k}} \frac{\partial Y_{k}}{\partial \tau}
$$

Now let $t=\tau, \quad Y_{0}(t)=t+t_{0}$, a linear function where the constant offset, $t_{0}$, maybe 0 , then $\partial Y_{0} / \partial t=1$

$$
\frac{d F\left(Y_{i}(t)\right)}{d t}=\sum_{k=0}^{6} \frac{\partial F}{\partial Y_{k}} \frac{\partial Y_{k}}{\partial t}
$$




\section{Javier Rivera}

By taking into account each variable contribution place brackets on the terms $\frac{\partial F}{\partial Y_{k}}$ to hold the labeled variables fixed provided by physical basis and for clarity.

For $\mathrm{j}=0$,

Need to fix both coordinates and the velocity field, since the partial time derivative is with respect to time

For $\mathrm{j}=1$ to 3 ,

$$
\frac{\partial F}{\partial Y_{0}} \frac{\partial Y_{0}}{\partial t}=\left[\frac{\partial \mathrm{F}(t, \vec{X}(t), \vec{u}(t, \vec{X}(t)))}{\partial \mathrm{t}}\right]_{\text {fixed } \vec{X} \& \vec{u}} 1
$$

Need to fix time since the partial derivative is with respect to the coordinates

For $\mathrm{i}=1$ to 3 ,

$$
\frac{\partial F}{\partial Y_{j}} \frac{\partial Y_{j}}{\partial t}=\left[\frac{\partial F(t, \vec{X}(t), \vec{u}(t, \vec{X}(t)))}{\partial X_{j}}\right]_{\text {fixed }} \frac{\partial X_{j}}{\partial t}
$$

Need to fix time since the partial derivative is with respect to the velocity field

$$
\frac{\partial F}{\partial Y_{i+3}} \frac{\partial Y_{i+3}}{\partial t}=\left[\frac{\partial F(t, \vec{X}(t), \vec{u}(t, \vec{X}(t)))}{\partial u_{i}}\right]_{\text {fixed } t} \frac{\partial u_{i}}{\partial t}
$$

Use the definition of the velocity field, $\frac{\partial X_{j}(t)}{\partial t}=\frac{d X_{j}(t)}{d t} \equiv u_{j}(t, \vec{X}(t))$, since the Eulerian coordinates are a function of time only, for Lagrangian velocity a partial time derivative on the Lagrangian coordinates is more applicable, $\frac{\partial x_{j}(t)}{\partial t} \equiv u_{j}\left(\vec{x}\left(t_{o}\right), t\right)$, results in the following definition of the field derivative of a field function, $F$, which was defined by a 7 dimensions directional derivative or "field" derivative from point $(\mathrm{t}, \boldsymbol{X}(t), \boldsymbol{U}(\mathrm{t}, \boldsymbol{X}(t)))$ to point $(\mathrm{t}+\Delta \mathrm{t}, \boldsymbol{X}(\mathrm{t}+\Delta \mathrm{t}), \boldsymbol{U}(\mathrm{t}+\Delta \mathrm{t}, \boldsymbol{X}(\mathrm{t}+\Delta \mathrm{t}))$ within a 1 -dimensional stream or curve in phasespace-time during the time interval, $\Delta$ t, as the time interval goes to 0 , where in the Taylor series we treat each variable as if they were "independent" coordinates of a point $(\mathrm{t}, \boldsymbol{X}(t)$, $\boldsymbol{U}(\mathrm{t}, \boldsymbol{X}(t)))$ within a curve in phase-space-time.

$$
\begin{aligned}
\frac{d}{d t} F(t, \vec{X}(t), \vec{u}( & t, \vec{X}(t))) \\
= & {\left[\frac{\partial \mathrm{F}(t, \vec{X}(t), \vec{u}(t, \vec{X}(t)))}{\partial \mathrm{t}}\right]_{\text {fixed } \vec{X}, \vec{u}} } \\
& +u_{j}(t, \vec{X}(t))\left[\frac{\partial F(t, \vec{X}(t), \vec{u}(t, \vec{X}(t)))}{\partial X_{j}}\right]_{\text {fixed } t} \\
& +\frac{\partial u_{i}(t, \vec{X}(t))}{\partial t}\left[\frac{\partial F(t, \vec{X}(t), \vec{u}(t, \vec{X}(t)))}{\partial u_{i}}\right]_{\text {fixed } t}
\end{aligned}
$$

The innovation in fluid mechanics, with this limit definition is that the dynamic changes of the fluid flow field are included in the tangent space of the field function, $F(t, \vec{X}(t), \vec{u}(\vec{X}, t))$, in the phase-space-time domain (7 dimensions). For elementary properties or rules of the field derivative, please see Section 2.1.

Q.E.D. 
On the Field Derivatives and its Application to Fluids

\section{Theorem 2. Fundamental Theorem of Calculus for the field derivative.}

If the time integral of field derivative of a field function $F(t, \vec{X}(t), \vec{u}(t, \vec{X}(t)))$ on a continues curve (or 1-dimensional stream) of field $\vec{u}(t, \vec{X}(t))$ from time 0 to time $\mathrm{t}$ is defined by the line integral

$$
\int_{0}^{t} \frac{d F(s, \vec{X}(s), \vec{u}(s, \vec{X}(s)))}{d s} d s
$$

Then the line integral result is given by the difference field function evaluated at the integral limits, $\mathrm{t}$ and 0 .

$$
\int_{0}^{t} \frac{d F(s, \vec{X}(s), \vec{u}(s, \vec{X}(s)))}{d s} d s=F(t, \vec{X}(t), \vec{u}(t, \vec{X}(t)))-F\left(0, \vec{X}(0), \vec{u}(0, \vec{X}(0))=\left.\Delta \mathrm{F}\right|_{0} ^{t}\right.
$$

Proof of Theorem 2. Fundamental Theorem of Calculus for the field derivative. By definition of the field derivative limit in Theorem 1,

$\frac{d F(t, \vec{X}(t), \vec{u}(t, \vec{X}(t)))}{d t}$

$$
\begin{aligned}
& \qquad \lim _{\Delta s \rightarrow 0} \frac{F(t+\Delta t, \vec{X}(t+\Delta t), \vec{u}(t+\Delta t, \vec{X}(t+\Delta t)))-F(t, \vec{X}(t), \vec{u}(t, \vec{X}(t)))}{\Delta t} \\
& \text { Let } t=t_{k-1}, \Delta t=t_{k}-t_{k-1}=\epsilon_{k} \\
& \begin{aligned}
\frac{d F(t, \vec{X}(t), \vec{u}(t, \vec{X}(t)))}{d t} \\
\quad=\lim _{\epsilon_{k} \rightarrow 0} \frac{F\left(t_{k}, \vec{X}\left(t_{k}\right), \vec{u}\left(t_{k}, \vec{X}\left(t_{k}\right)\right)\right)-F\left(t_{k-1}, \vec{X}\left(t_{k-1}\right), \vec{u}\left(t_{k-1}, \vec{X}\left(t_{k-1}\right)\right)\right)}{\epsilon_{k}}
\end{aligned}
\end{aligned}
$$

From the definition of a line integral [5], thus, each point in the line integral is in a continues curve of the field (or 1-dimensional stream of the fluid velocity)

$$
\begin{aligned}
& \int_{0}^{t} \frac{d F(s, \vec{X}(s), \vec{u}(s, \vec{X}(s)))}{d s} d s \\
& =\lim _{n \rightarrow \infty} \sum_{k=1}^{(n+1)} \lim _{\epsilon_{k} \rightarrow 0} \frac{F\left(t_{k}, \vec{X}\left(t_{k}\right), \vec{u}\left(t_{k}, \vec{X}\left(t_{k}\right)\right)\right)-F\left(t_{k-1}, \vec{X}\left(t_{k-1}\right), \vec{u}\left(t_{k-1}, \vec{X}\left(t_{k-1}\right)\right)\right)}{\epsilon_{k}} \epsilon_{k}
\end{aligned}
$$

The sum is finite; the epsilon limit commutes with the finite summation,

$$
\begin{aligned}
& \int_{0}^{t} \frac{d F(s, \vec{X}(s), \vec{u}(s, \vec{X}(s)))}{d s} d s \\
& \quad=\lim _{n \rightarrow \infty} \lim _{\epsilon_{k} \rightarrow 0} \sum_{k=1}^{(n+1)} F\left(t_{k}, \vec{X}\left(t_{k}\right), \vec{u}\left(t_{k}, \vec{X}\left(t_{k}\right)\right)\right)-F\left(t_{k-1}, \vec{X}\left(t_{k-1}\right), \vec{u}\left(t_{k-1}, \vec{X}\left(t_{k-1}\right)\right)\right)
\end{aligned}
$$

Summing the finite sum, cancelling terms out, and taking the epsilon limit. As $n \rightarrow$ $\infty$, with $t_{k}-t_{k-1}=\epsilon_{k} \rightarrow 0$, as $\bar{\epsilon} \rightarrow 0$ thus, the $\operatorname{limit}_{n \rightarrow \infty} t_{n+1}=\operatorname{limit}_{n \rightarrow \infty}(n+1) \bar{\epsilon}=t$ converges by construction. 


\section{Javier Rivera}

$$
\begin{aligned}
& \int_{0}^{t} \frac{d F(s, \vec{X}(s), \vec{u}(s, \vec{X}(s)))}{d s} d s \\
& \quad=\lim _{n \rightarrow \infty} F\left(t_{n+1}, \vec{X}\left(t_{n+1}\right), \vec{u}\left(t_{n+1}, \vec{X}\left(t_{n+1}\right)\right)\right)-F\left(t_{0}, \vec{X}\left(t_{0}\right), \vec{u}\left(t_{0}, \vec{X}\left(t_{0}\right)\right)\right)
\end{aligned}
$$

Define $t_{0}=0$. Thus, the line integral along a 1-dimensional stream or curve result is given by the difference field function evaluated at the integral limits. (See Section 3.3 for a more complete proof with the induction method).

$$
\begin{aligned}
& \int_{0}^{t} \frac{d F(s, \vec{X}(s), \vec{u}(s, \vec{X}(s)))}{d s} d s=F(t, \vec{X}(t), \vec{u}(t, \vec{X}(t)))-F(0, \vec{X}(0), \vec{u}(0, \vec{X}(0)))=\left.\Delta F\right|_{0} ^{t} \\
& \text { Q.E.D. }
\end{aligned}
$$

\subsection{Examples of field derivative}

Example 1. Field derivative of the fluid velocity field.

Let $F_{k}(t, \vec{X}(t), \vec{u}(t, \vec{X}(t)))=u_{k}(t, \vec{X}(t))$, the fluid velocity field, then

- $\left[\frac{\partial \mathrm{F}_{k}(t, \vec{X}(t), \vec{u}(t, \vec{X}(t)))}{\partial \mathrm{t}}\right]_{\text {fixed } \vec{X}, \vec{u}}=0$

- $u_{j}(t, \vec{X}(t))\left[\frac{\partial F_{k}(t, \vec{X}(t), \vec{u}(t, \vec{X}(t)))}{\partial X_{j}}\right]_{f i x e d ~}=u_{j}(t, \vec{X}(t)) \frac{\partial u_{k}(t, \vec{X}(t))}{\partial X_{j}}$

- $\quad \frac{\partial u_{i}(t, \vec{X}(t))}{\partial t}\left[\frac{\partial F_{k}(t, \vec{X}(t), \vec{u}(t, \vec{X}(t)))}{\partial u_{i}}\right]_{\text {fixed } t}=\frac{\partial u_{i}(t, \vec{X}(t))}{\partial t} \frac{\partial u_{k}(t, \vec{X}(t))}{\partial u_{i}}=\frac{\partial u_{i}(t, \vec{X}(t))}{\partial t} \cdot \delta_{k, i}=\frac{\partial u_{k}(t, \vec{X}(t))}{\partial t}$

where $\delta_{k, i}$ is the Kronecker Delta discrete function. Thus, the mathematical definition of the field derivative of the fluid velocity vector gives same result as the material derivative fluid velocity

$$
\frac{d u_{k}(t, \vec{X}(t))}{d t}=0+u_{j}(t, \vec{X}(t)) \frac{\partial u_{k}(t, \vec{X}(t))}{\partial X_{j}}+\frac{\partial u_{k}(t, \vec{X}(t))}{\partial t}=\frac{D u_{k}(t, \vec{X}(t))}{D t}
$$

This proves the statement that the field derivative defined in the phase-space-time is identical to the material derivative as defined by Ivanova et al [6].

Example 2. Field derivative of Eulerian space coordinates.

Let $F_{k}(t, \vec{X}(t), \vec{u}(t, \vec{X}(t)))=\mathrm{X}_{k}(\mathrm{t})$, a function of Eulerian space coordinate

- $\left[\frac{\partial \mathrm{F}_{k}(t, \vec{X}(t), \vec{u}(t, \vec{X}(t)))}{\partial \mathrm{t}}\right]_{\text {fixed } \vec{x}, \vec{u}}=0$

- $u_{j}(t, \vec{X}(t))\left[\frac{\partial F_{k}(t, \vec{X}(t), \vec{u}(t, \vec{X}(t)))}{\partial X_{j}}\right]_{\text {fixed } t}=u_{j}(t, \vec{X}(t)) \frac{\partial}{\partial X_{j}} \mathrm{X}_{k}(\mathrm{t})=u_{j}(t, \vec{X}(t)) \delta_{k, j}=$

$$
u_{k}(t, \vec{X}(t))
$$

- $\quad \frac{\partial u_{i}(t, \vec{X}(t))}{\partial t}\left[\frac{\partial F_{k}(t, \vec{X}(t), \vec{u}(t, \vec{X}(t)))}{\partial u_{i}}\right]_{\text {fixed } t}=\frac{\partial u_{i}(t, \vec{X}(t))}{\partial t} \cdot 0=0$

Thus, the result is identical to the elementary time differentiation. 
On the Field Derivatives and its Application to Fluids

$$
\frac{d \mathrm{X}_{k}(\mathrm{t})}{d t}=u_{k}(t, \vec{X}(t))
$$

Example 3. Field derivative of a tensor density field.

$$
\begin{aligned}
& \text { Let } F_{k, m}(t, \vec{X}(t), \vec{u}(t, \vec{X}(t)))=\frac{\partial u_{k}(t, \vec{X}(t))}{\partial X_{m}}
\end{aligned}
$$

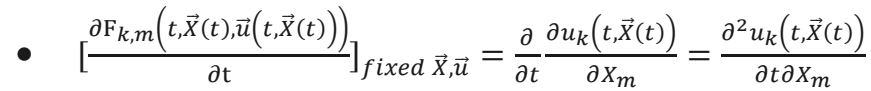

$$
\begin{aligned}
& \text { - } u_{j}(t, \vec{X}(t))\left[\frac{\partial F_{k, m}(t, \vec{X}(t) \vec{u}(t, \vec{X}(t)))}{\partial X_{j}}\right]_{\text {fixed } t}=u_{j}(t, \vec{X}(t)) \frac{\partial}{\partial X_{j}} \frac{\partial u_{k}(t, \vec{X}(t))}{\partial X_{m}}= \\
& u_{j}(t, \vec{X}(t)) \frac{\partial^{2} u_{k}(t, \vec{X}(t))}{\partial X_{j} \partial X_{m}} \\
& \text { - } \quad \frac{\partial u_{i}(t, \vec{X}(t))}{\partial t}\left[\frac{\partial F_{k}(t, \vec{X}(t), \vec{u}(t, \vec{X}(t)))}{\partial u_{i}}\right]_{f i x e d ~}=\frac{\partial u_{i}(t, \vec{X}(t))}{\partial t} \cdot \frac{\partial}{\partial X_{m}} \frac{\partial u_{k}(t, \vec{X}(t))}{\partial u_{i}}=\frac{\partial u_{i}(t, \vec{X}(t))}{\partial t} \cdot \frac{\partial}{\partial X_{m}} \delta_{k, i}=0 \\
& \frac{d F_{k, m}}{d t}=\frac{d}{d t} \frac{\partial u_{k}(t, \vec{X}(t))}{\partial X_{m}}=\frac{\partial^{2} u_{k}(t, \vec{X}(t))}{\partial t \partial X_{m}}+u_{j}(t, \vec{X}(t)) \frac{\partial^{2} u_{k}(t, \vec{X}(t))}{\partial X_{j} \partial X_{m}}
\end{aligned}
$$

Example 4. Field derivative product rule

$$
\begin{gathered}
\frac{d F(t, \vec{X}(t), \vec{u}(t, \vec{X}(t))) G(t, \vec{X}(t), \vec{u}(t, \vec{X}(t)))}{d t} \\
=\lim _{\Delta t \rightarrow 0} \frac{F(t+\Delta t, \vec{X}(t+\Delta t), \vec{u}(t+\Delta t, \vec{X}(t+\Delta t))) G(t+\Delta t, \vec{X}(t+\Delta t), \vec{u}(t+\Delta t, \vec{X}(t+\Delta t)))}{\Delta t} \\
=\lim _{\Delta t \rightarrow 0}\left[\frac{1}{\Delta t}(F(t+\Delta t, \vec{X}(t+\Delta t), \vec{u}(t+\Delta t, \vec{X}(t+\Delta t))) G(t+\Delta t, \vec{X}(t+\Delta t), \vec{u}(t+\Delta t, \vec{X}(t+\Delta t)))\right. \\
-F(t, \vec{X}(t), \vec{u}(t, \vec{X}(t))) G(t+\Delta t, \vec{X}(t+\Delta t), \vec{u}(t+\Delta t, \vec{X}(t+\Delta t))) \\
+\frac{1}{\Delta t}(F(t, \vec{X}(t), \vec{u}(t, \vec{X}(t))) G(t+\Delta t, \vec{X}(t+\Delta t), \vec{u}(t+\Delta t, \vec{X}(t+\Delta t))) \\
-F(t, \vec{X}(t), \vec{u}(t, \vec{X}(t))) G(t, \vec{X}(t), \vec{u}(t, \vec{X}(t))))] \\
=\frac{d F(t, \vec{X}(t), \vec{u}(t, \vec{X}(t)))}{d t} G(t, \vec{X}(t), \vec{u}(t, \vec{X}(t))) \\
+F(t, \vec{X}(t), \vec{u}(t, \vec{X}(t))) \frac{d G(t, \vec{X}(t), \vec{u}(t, \vec{X}(t)))}{d t}
\end{gathered}
$$




\section{Javier Rivera}

\section{Example 5. Field derivative Integration by Parts}

$\int_{0}^{t} u_{i}(s, \vec{X}(s)) \frac{d \delta u_{i}(s, \vec{X}(s))}{d s} d s=\left.u_{i}(s, \vec{X}(s)) \delta u_{i}(s, \vec{X}(s))\right|_{0} ^{t}-\int_{0}^{t} \frac{d u_{i}(s, \vec{X}(s))}{d s} \delta u_{i}(s, \vec{X}(s)) d s$

Proof:

$$
\begin{aligned}
& \int_{0}^{t} \frac{d\left(u_{i}(s, \vec{X}(s)) \delta u_{i}(s, \vec{X}(s))\right)}{d s} d s=\left.u_{i}(s, \vec{X}(s)) \delta u_{i}(s, \vec{X}(s))\right|_{0} ^{t} \text { by Theorem } 2 \\
& \int_{0}^{t} \frac{d\left(u_{i}(s, \vec{X}(s))\right)}{d s} \delta u_{i}(s, \vec{X}(s))+\frac{d u_{i}(s, \vec{X}(s))}{d s} \delta u_{i}(s, \vec{X}(s)) d s=\left.u_{i}(s, \vec{X}(s)) \delta u_{i}(s, \vec{X}(s))\right|_{0} ^{t}
\end{aligned}
$$

by Theorem 1, and differentiation product rule, example \#4. Rearranging gives

$$
\int_{0}^{t} u_{i}(s, \vec{X}(s)) \frac{d \delta u_{i}(s, \vec{X}(s))}{d s} d s=\left.u_{i}(s, \vec{X}(s)) \delta u_{i}(s, \vec{X}(s))\right|_{0} ^{t}-\int_{0}^{t} \frac{d u_{i}(s, \vec{X}(s))}{d s} \delta u_{i}(s, \vec{X}(s)) d s
$$

Example 6. Integral of the field derivative of the Lagrangian Bernoulli Scalar Field

For the scalar field example use the Lagrangian Bernoulli formula. Time integral of the field derivative of the Lagrangian Bernoulli scalar field,

$$
\int_{0}^{t} \frac{d B(s, \vec{x}(s), \vec{u}(s, \vec{x}(s)))}{d s} d s=B(t, \vec{x}(t), \vec{u}(t, \vec{x}(t)))-B\left(0, \vec{x}(0), \vec{u}(0, \vec{x}(0))=\left.\Delta \mathrm{B}\right|_{0} ^{t}\right.
$$

The change in total mechanical energy per unit mass of the fluid in the stream cylindrical control volume, $\delta V_{k-1}$, is the volume integral below in Eulerian coordinates. Where the Jacobian for k and k-1 times are the same or are constant equal to unity due to fluids incompressibility. Since the total mechanical energy is the same for the stream line for small interval of times then,

$$
\begin{aligned}
E_{T}\left(t_{k}\right)-E_{T}\left(t_{k-1}\right) & \\
& =\iiint_{\delta V_{k-1}}\left[B\left(t_{k}, \vec{x}\left(t_{k}\right), \vec{u}\left(t_{k}, \vec{x}\left(t_{k}\right)\right)\right)\right. \\
& \left.-B\left(t_{k-1}, \vec{x}\left(t_{k-1}\right), \vec{u}\left(t_{k-1}, \vec{x}\left(t_{k-1}\right)\right)\right)\right] \rho_{o} \frac{\partial\left(x_{1}, x_{2}, x_{3}\right)}{\partial\left(R_{1}, R_{2}, R_{3}\right)} d V=0
\end{aligned}
$$

By taking the limit as the radius of the cylinder control volume goes to zero, $\delta V_{k-1} \rightarrow 0$.

The length of the cylinder only diminishes with increasing number of samples.

Since $\rho_{o} \frac{\partial\left(x_{1}, x_{2}, x_{3}\right)}{\partial\left(R_{1}, R_{2}, R_{3}\right)}>0$, thus,

$$
\begin{aligned}
\lim _{\delta V_{k-1} \rightarrow 0} \frac{1}{\delta V_{k-1}} & \iiint_{\delta V_{k-1}}\left[B\left(t_{k}, \vec{x}\left(t_{k}\right), \vec{u}\left(t_{k}, \vec{x}\left(t_{k}\right)\right)\right)\right. \\
& \left.-B\left(t_{k-1}, \vec{x}\left(t_{k-1}\right), \vec{u}\left(t_{k-1}, \vec{x}\left(t_{k-1}\right)\right)\right)\right] \rho_{o} \frac{\partial\left(x_{1}, x_{2}, x_{3}\right)}{\partial\left(R_{1}, R_{2}, R_{3}\right)} d V=0
\end{aligned}
$$


On the Field Derivatives and its Application to Fluids

$$
B\left(t_{k}, \vec{x}\left(t_{k}\right), \vec{u}\left(\vec{x}\left(t_{k}\right), t_{k}\right)\right)-B\left(t_{k-1}, \vec{x}\left(t_{k-1}\right), \vec{u}\left(t_{k-1}, \vec{x}\left(t_{k-1}\right)\right)\right)=0
$$

where the Eulerian Bernoulli formula is defined below for $\mathrm{k}=1$, small time $t_{l}$,

$$
\begin{aligned}
B\left(t_{1}, \vec{x}\left(t_{1}\right), \vec{u}\left(t_{1}, \vec{x}\left(t_{1}\right)\right)\right)-B\left(t_{0}, \vec{x}\left(t_{0}\right), \vec{u}\left(t_{0}, \vec{x}\left(t_{0}\right)\right)\right. & \left(\frac{1}{2} u_{i}^{2}\left(t_{1}, \vec{x}\left(t_{1}\right)\right)-\frac{1}{2} u_{i}^{2}\left(t_{0}, \vec{x}\left(t_{0}\right)\right)+\phi\left(\vec{x}\left(t_{1}\right)\right)-\phi\left(\vec{x}\left(t_{0}\right)\right)+\frac{p\left(\vec{x}\left(t_{1}\right)\right)}{\rho_{o}}\right. \\
& \left.-\frac{p\left(\vec{x}\left(t_{0}\right)\right)}{\rho_{o}}+\frac{1}{\rho_{o}} \int_{t_{0}}^{t_{1}} \tau_{i, j} \frac{\partial u_{i}}{\partial x_{j}} d s+I_{o}\right)=0
\end{aligned}
$$

Thus, for simplicity, let $V(\vec{x}(t))=\frac{p(\vec{x}(t))}{\rho_{o}}+\phi(\vec{x}(t))$ to obtain the difference sample Bernoulli equations for each difference $\mathrm{k}$.

$$
\begin{aligned}
B\left(t_{k}, \vec{x}\left(t_{k}\right), \vec{u}\left(t_{k}, \vec{x}\left(t_{k}\right)\right)\right)-B\left(t_{k-1}, \vec{x}\left(t_{k-1}\right), \vec{u}\left(t_{k-1}, \vec{x}\left(t_{k-1}\right)\right)\right) \\
=\frac{1}{2} u_{i}^{2}\left(t_{k}, \vec{x}\left(t_{k}\right)\right)-\frac{1}{2} u_{i}^{2}\left(t_{k-1}, \vec{x}\left(t_{k-1}\right)\right)+v\left(\vec{x}\left(t_{k}\right)\right)-v\left(\vec{x}\left(t_{k-1}\right)\right)+\frac{1}{\rho_{o}} \int_{t_{k-1}}^{t_{k}} \tau_{i, j} \frac{\partial u_{i}}{\partial x_{j}} d s
\end{aligned}
$$

By the proof of Theorem 2 Fundamental Theorem of calculus and proof by induction in Section 3.3, thus,

$$
\int_{0}^{t} \frac{d B(s, \vec{x}(s), \vec{u}(s, \vec{x}(s)))}{d s} d s=B(t, \vec{x}(t), \vec{u}(t, \vec{x}(t)))-B(0, \vec{x}(0), \vec{u}(0, \vec{x}(0)))=\left.\Delta B\right|_{0} ^{t}
$$

See Section 3.2 for more details on the derivation of the Bernoulli principle using the field derivative. Although the functional argument notation used in sections 3.0, 3.1, and 3.3 is the same as Appendix A, i.e. the velocity field is implicit and the fluid velocity functional arguments are in reverse order to match reference [1].

\section{Derivation of Bernoulli's principle for incompressible viscous fluids}

The internal energy per unit mass, $I(t)$, of the fluid was defined in article [1], as shown in (Eq. 1).

$$
I(t)=\frac{1}{\rho_{o}} \int_{0}^{t} \tau_{i, j} \frac{\partial u_{i}}{\partial X_{j}} d s+I_{o}
$$

Equations 4.2, 4.3 and 4.4 in the original article [1] are reproduced below as equations 2, 3 , and 4 , respectively, with the change being that the dissipation functional definition ${ }^{1}$ is used for the convenience of the reader, where the Einstein summation convention is used throughout this manuscript with indices $\mathrm{i}$ and $\mathrm{j}$ equal to 1,2 and 3.

The Hamiltonian per unit mass was defined as shown in (Eq. 2):

$$
H=K+V-\frac{1}{2} I(t)=\frac{1}{2} u_{i}^{2}+\phi(\vec{X})+\frac{p(\vec{X})}{\rho_{o}}-\frac{1}{2 \rho_{o}} \int_{0}^{t} \tau_{i, j} \frac{\partial u_{i}}{\partial X_{j}} d s-\frac{1}{2} I_{o}
$$

where $K, V, I(t), \rho_{o}, V_{\mathrm{o}}, \phi, p, u_{i}, \tau_{i, j}, X_{j}$, and $I_{o}$ are the kinetic energy, potential energy, internal energy, constant fluid density, constant volume, external potential energy, fluid pressure, velocity of the fluid, viscous stress tensor, Eulerian $\mathrm{j}^{\text {th }}$ spatial coordinate, and

\footnotetext{
${ }^{1}$ The dissipation function, $D$, can be defined as $D=\tau_{i, j} \frac{\partial u_{i}}{\partial x_{j}}$ (equation 2.3 in reference [1]). The fluid's internal energy is given by $I(t)=\frac{1}{\rho_{o}} \int_{0}^{t} \tau_{i, j} \frac{\partial u_{i}}{\partial x_{j}} d s+I_{o}$.
} 


\section{Javier Rivera}

constant fluid mechanical internal energy per unit mass, respectively. The time rate of change $^{2}$ of the Hamiltonian is given in (Eq. 3).

$$
\frac{d H}{d t}=\frac{d}{d t}(K+V)-\frac{1}{2} \frac{d I}{d t}=-\frac{3}{2} \frac{d I}{d t}
$$

The total mechanical energy per unit mass, $E_{T}$, is conserved, as shown in (Eq. 4).

$$
\frac{d}{d t}\left(H+\frac{3}{2} I\right)=\frac{d}{d t}(K+V+I)=\frac{d E_{T}}{d t}=0
$$

This derivation appeared to be an interesting mathematical puzzling result in the sense that the time integral might diverge; thus, the question arises as to how it could remain a constant. The solution to this puzzle is because the volume integrals were implicit in (Eq. 2), (Eq. 3), and (Eq. 4). Additionally, the time differentiation of the total mechanical energy was expected to be the material derivative, yet this puzzle has generated an idea of the field derivative. Yet, the following subsection is based on Appendix A material derivative method published in reference [6].

\subsection{Derivation of Bernoulli's principle for incompressible viscous fluids using material derivative method}

Therefore, the total mechanical energy, $E_{T}$, is given by (Eq. 5).

$$
E_{T}(t)=\iiint_{\delta V_{o}}\left(\frac{1}{2} u_{i}^{2}+\phi(\vec{X})+\frac{p(\vec{X})}{\rho_{o}}+\frac{1}{\rho_{o}} \int_{0}^{t} \tau_{i, j} \frac{\partial u_{i}}{\partial X_{j}} d s+I_{o}\right) \rho_{o} \frac{\partial\left(X_{1}, X_{2}, X_{3}\right)}{\partial\left(R_{1}, R_{2}, R_{3}\right)} d V_{o}
$$

The volume integral has a finite stream control volume, $\delta V_{o}$, which encloses the fluid motion of fluid stream flow (for example, like a cylinder of fixed length, $\delta L$, and small radius, $\delta r_{o}$, to encompass a small portion of a moving stream within a river or ocean) from the initial time, 0 , to some small time, t, for a fixed small length $\delta L$.

In performing the material derivative [6] to the total mechanical energy by integration by parts on the viscous stress tensor term, where the resulting surface integral is zero $\left(\oiint_{\delta S_{o}} \tau_{i, j} u_{i} n_{j} \rho_{o} d S=0\right)$ via the nonslip condition for solid boundaries or require the fluid shear stress to be null because the surface normal may be arbitrary in absence of solid boundaries, results in (Eq. 6) (See Appendix A). Since $\frac{D}{D t}\left(\rho_{o} \frac{\partial\left(X_{1}, X_{2}, X_{3}\right)}{\partial\left(R_{1}, R_{2}, R_{3}\right)}\right)=0$, by fluid continuity equation and using Reynolds Transport Theorem (incompressible condition can be relaxed), or $\frac{\partial\left(X_{1}, X_{2}, X_{3}\right)}{\partial\left(R_{1}, R_{2}, R_{3}\right)}=1$ for incompressible fluids.

$$
\frac{D}{D t} E_{T}(t)=\iiint_{\delta V_{o}} u_{i}\left(\frac{\partial u_{i}}{\partial t}+u_{j} \frac{\partial u_{i}}{\partial X_{j}}+\frac{\partial \phi}{\partial X_{i}}+\frac{1}{\rho_{o}} \frac{\partial p}{\partial X_{i}}-\frac{1}{\rho_{o}} \frac{\partial \tau_{i, j}}{\partial X_{j}}\right) \rho_{o} \frac{\partial\left(X_{1}, X_{2}, X_{3}\right)}{\partial\left(R_{1}, R_{2}, R_{3}\right)} d V_{o}=0
$$

The terms inside the parenthesis, which are the Navier-Stokes equations for incompressible fluids, vanish throughout the fluid for all coordinates $\vec{X}(t)$ within control volume, $V_{o}$; thus, the fluid's total mechanical energy is a constant, $E_{\mathrm{o}}$.

$$
E_{T}\left(t_{1}\right)=\iiint_{\delta V_{o}}\left(\frac{1}{2} u_{i}^{2}+\phi(\vec{X})+\frac{p(\vec{X})}{\rho_{o}}+\frac{1}{\rho_{o}} \int_{0}^{t_{1}} \tau_{i, j} \frac{\partial u_{i}}{\partial X_{j}} d s+I_{o}\right) \rho_{o} \frac{\partial\left(X_{1}, X_{2}, X_{3}\right)}{\partial\left(R_{1}, R_{2}, R_{3}\right)} d V_{o}=E_{o}
$$

Define $E_{o}=B_{o} \rho_{o} V_{o}$ where $B_{o}$ is a constant mechanical energy per unit mass. Note that time $\mathrm{t}_{1}$ is short.

\footnotetext{
${ }^{2}$ Time rate of change implied in this manuscript and the in reference [1] is the material derivative as defined by Batchelor [7] and reference [6].
} 
On the Field Derivatives and its Application to Fluids

$$
\begin{gathered}
\iiint_{\delta V_{o}}\left(\frac{1}{2} u_{i}^{2}+\phi(\vec{X})+\frac{p(\vec{X})}{\rho_{o}}+\frac{1}{\rho_{o}} \int_{0}^{t_{1}} \tau_{i, j} \frac{\partial u_{i}}{\partial X_{j}} d s+I_{o}\right) \rho_{o} \frac{\partial\left(X_{1}, X_{2}, X_{3}\right)}{\partial\left(R_{1}, R_{2}, R_{3}\right)} d V_{o} \\
=\iiint_{\delta V_{o}}\left(B_{o} \rho_{o} \frac{\partial\left(X_{1}, X_{2}, X_{3}\right)}{\partial\left(R_{1}, R_{2}, R_{3}\right)}\right) d V_{o}
\end{gathered}
$$

Therefore, the Bernoulli's principle is given as in (Eq. 7).

$$
\iiint_{\delta V_{o}}\left(\frac{1}{2} u_{i}^{2}+\phi(\vec{X})+\frac{p(\vec{X})}{\rho_{o}}+\frac{1}{\rho_{o}} \int_{0}^{t_{1}} \tau_{i, j} \frac{\partial u_{i}}{\partial X_{j}} d s+I_{o}-B_{o}\right) \rho_{o} \frac{\partial\left(X_{1}, X_{2}, X_{3}\right)}{\partial\left(R_{1}, R_{2}, R_{3}\right)} d V_{o}=0
$$

The integrand in (Eq. 7) must be zero for all Eulerian coordinates, $\vec{X}$, within the stream control volume and small times, $1>\mathrm{t} \geq 0$. Next, the control volume, $V_{o}$, containing the stream or current is shrunk to zero volume, i.e. from 3 dimensions to 1 dimension. For example, let the radius, $\delta r_{o}$, of a flexible tube control volume go to zero for every current or stream in the control volume, the flexible tube tends to a 1-dimensional stream; Thus, taking the limit as the volume goes to zero results in the integrand of (Eq. $7 A$ ) being zero at every point of the 1-dimensional stream, therefore $(E q .7 B)$ is valid throughout the fluid with different values of $B_{o}$, by partitioning the fluid flow with control volumes containing them. The proof requires a multiple use (by induction) of the Mean Value Theorem of Integrals for every point in the stream, see footnote in page 71 of [2].

limit $_{\delta V_{o} \rightarrow 0} \frac{1}{\rho_{o} \delta V_{o}} \iiint_{V_{o}}\left(\frac{1}{2} u_{i}^{2}+\phi(\vec{X})+\frac{p(\vec{X})}{\rho_{o}}+\frac{1}{\rho_{o}} \int_{0}^{t} \tau_{i, j} \frac{\partial u_{i}}{\partial X_{j}} d s+I_{o}-B_{o}\right) \rho_{o} \frac{\partial\left(X_{1}, X_{2}, X_{3}\right)}{\partial\left(R_{1}, R_{2}, R_{3}\right)} d V_{o}=0$

Since $\rho_{o} \frac{\partial\left(X_{1}, X_{2}, X_{3}\right)}{\partial\left(R_{1}, R_{2}, R_{3}\right)}>0$, the limit of $(E q .7 A)$ yields the Eulerian Bernoulli equation for viscous incompressible fluids in $(E q .7 B)$ within a 1-dimensional stream.

$$
B(\vec{X}(t), t)=\frac{1}{2} u_{i}^{2}(\vec{X}(t), t)+\phi(\vec{X}(t))+\frac{p(\vec{X}(t))}{\rho_{o}}+\frac{1}{\rho_{o}} \int_{0}^{t} \tau_{i, j} \frac{\partial u_{i}}{\partial X_{j}} d s+I_{o}=B_{o}
$$

Equations (Eqs. $7 A-B$ ) remain valid when $\vec{X}(t) \rightarrow \vec{X}_{o}$ as $t \rightarrow 0$. The constant $B_{o}$ is identified with the initial conditions found in $(E q .7 C)$ valid in the 1-dimensional stream.

$$
\text { Eulerian, } \frac{1}{2} u_{i}^{2}\left(\vec{X}_{o}, 0\right)+\phi\left(\vec{X}_{o}\right)+\frac{p\left(\vec{X}_{o}\right)}{\rho_{o}}+I_{o}=B_{o}
$$

\subsection{Derivation of Bernoulli's principle for incompressible viscous fluids using the field derivative}

This section demonstrates an alternate derivation using Section 2 mathematical methods of field derivative without using Appendix A definitions of material derivative.

$$
\begin{aligned}
B(t, \vec{X}(t), \vec{u}(t, \vec{X}(t))=( & \left.\frac{1}{2} u_{i}^{2}(t, \vec{X}(t))+\phi(\vec{X})+\frac{p(\vec{X})}{\rho_{o}}+\frac{1}{\rho_{o}} \int_{0}^{t} \tau_{i, j} \frac{\partial u_{i}}{\partial X_{j}} d s+I_{o}\right) \\
\frac{d}{d t} B(t, \vec{X}(t), \vec{u}( & t, \vec{X}(t))) \\
= & {\left[\frac{\partial \mathrm{B}(t, \vec{X}(t), \vec{u}(t, \vec{X}(t)))}{\partial \mathrm{t}]_{\text {fixed } \vec{X}, \vec{u}}}\right.} \\
& +u_{j}(t, \vec{X}(t))\left[\frac{\partial B(t, \vec{X}(t), \vec{u}(t, \vec{X}(t)))}{\partial X_{j}}\right]_{\text {fixed } t} \\
& +\frac{\partial u_{i}(t, \vec{X}(t))}{\partial t}\left[\frac{\partial B(t, \vec{X}(t), \vec{u}(t, \vec{X}(t)))}{\partial u_{i}}\right]_{\text {fixed } t}
\end{aligned}
$$




\section{Javier Rivera}

Partial differentiating each term and holding fixed the variables in the vertical bar or bracket

$$
\begin{gathered}
\left.\frac{\partial \mathrm{B}(t, \vec{X}(t), \vec{u}(t, \vec{X}))}{\partial \mathrm{t}}\right|_{\text {fixed } \vec{X}, \vec{u}}=\frac{1}{\rho_{o}} \tau_{i, j} \frac{\partial u_{i}}{\partial X_{j}} ; \\
u_{j}(t, \vec{X}(t))\left[\frac{\partial B(t, \vec{X}(t), \vec{u}(t, \vec{X}))}{\partial X_{j}}\right]_{\text {fixed } t} \\
=u_{j}(t, \vec{X}(t)) \frac{\partial}{\partial X_{j}} \phi(\vec{X})+u_{j}(t, \vec{X}(t)) \frac{\partial}{\partial X_{j}} \frac{p(\vec{X})}{\rho_{o}} \\
+u_{j}(t, \vec{X}(t)) \frac{\partial u_{i}}{\partial X_{j}} u_{i}(t, \vec{X}(t))
\end{gathered}
$$

Using chain rule in the last term,

$$
\begin{gathered}
\frac{\partial u_{i}(t, \vec{X}(t))}{\partial t}\left[\frac{\partial B(t, \vec{X}(t), \vec{u}(t, \vec{X}))}{\partial u_{i}}\right]_{\text {fixed } t}=\frac{\partial u_{i}(t, \vec{X}(t))}{\partial t} u_{i}(t, \vec{X}(t)) \\
=u_{i}(t, \vec{X}(t)) \frac{\partial u_{i}(t, \vec{X}(t))}{\partial t}
\end{gathered}
$$

Thus, adding all the terms the field derivative,

$\frac{d}{d t} B(t, \vec{X}(t), \vec{u}(t, \vec{X}))=$

$\frac{1}{\rho_{o}} \tau_{i, j} \frac{\partial u_{i}}{\partial X_{j}}+u_{j}(t, \vec{X}(t)) \frac{\partial}{\partial X_{j}} \phi(\vec{X})+u_{j}(t, \vec{X}(t)) \frac{\partial}{\partial X_{j}} \frac{p(\vec{X})}{\rho_{o}}+u_{i}(t, \vec{X}(t)) u_{j}(t, \vec{X}(t)) \frac{\partial u_{i}}{\partial X_{j}}+$

$u_{i}(t, \vec{X}(t)) \frac{\partial u_{i}(t, \vec{X}(t))}{\partial t}$

Changing dummy index from $\mathrm{j}$ to $\mathrm{i}$ on the potential energy terms

$\frac{d}{d t} B(t, \vec{X}(t), \vec{u}(t, \vec{X}(t)))=\frac{1}{\rho_{o}} \tau_{i, j} \frac{\partial u_{i}}{\partial X_{j}}+u_{i}(t, \vec{X}(t)) \frac{\partial}{\partial X_{i}} \phi(\vec{X})+u_{i}(t, \vec{X}(t)) \frac{\partial}{\partial X_{i}} \frac{p(\vec{X})}{\rho_{o}}+$

$u_{i}(t, \vec{X}(t)) u_{j}(t, \vec{X}(t)) \frac{\partial u_{i}}{\partial x_{j}}+u_{i}(t, \vec{X}(t)) \frac{\partial u_{i}(t, \vec{X}(t))}{\partial t}$

Rearranging the terms to obtain

$\frac{d}{d t} B(t, \vec{X}(t), \vec{u}(t, \vec{X}(t)))=u_{i}(t, \vec{X}(t))\left[\frac{\partial u_{i}(t, \vec{X}(t))}{\partial t}+\frac{\partial}{\partial X_{i}} \phi(\vec{X})+\frac{\partial}{\partial X_{i}} \frac{p(\vec{X})}{\rho_{o}}+u_{j}(t, \vec{X}(t)) \frac{\partial u_{i}}{\partial X_{j}}\right]+$ $\frac{1}{\rho_{o}} \tau_{i, j} \frac{\partial u_{i}}{\partial X_{j}}$

Since for small time intervals, $\Delta t$, since the cylindrical control volume is nearly fixed volume, or since the fluid is incompressible (the Jacobian is nearly constant,

$$
\left.\frac{\partial\left(X_{1}, X_{2}, X_{3}\right)}{\partial\left(R_{1}, R_{2}, R_{3}\right)} \sim 1\right) .
$$

By integration by parts of the shear stress term, assuming the required surface boundary condition, where either the fluid velocity has a no-slip condition or the surface normal is arbitrary; therefore, the shear stress is zero at the volume surface, as expected. 
On the Field Derivatives and its Application to Fluids

$$
\begin{gathered}
\iiint_{\delta V_{o}} \frac{1}{\rho_{o}} \tau_{i, j} \frac{\partial u_{i}}{\partial X_{j}} \rho_{o} \frac{\partial\left(X_{1}, X_{2}, X_{3}\right)}{\partial\left(R_{1}, R_{2}, R_{3}\right)} d V_{o}=\oiint_{\delta S_{o}} \tau_{i, j} u_{i} n_{j} d S-\iiint_{\delta V_{o}} \frac{1}{\rho_{o}} \frac{\partial \tau_{i, j}}{\partial X_{j}} u_{i} \rho_{o} \frac{\partial\left(X_{1}, X_{2}, X_{3}\right)}{\partial\left(R_{1}, R_{2}, R_{3}\right)} d V_{o} \\
=-\iiint_{\delta V_{o}} \frac{1}{\rho_{o}} \frac{\partial \tau_{i, j}}{\partial X_{j}} u_{i} \rho_{o} \frac{\partial\left(X_{1}, X_{2}, X_{3}\right)}{\partial\left(R_{1}, R_{2}, R_{3}\right)} d V_{o}
\end{gathered}
$$

Therefore, the field derivative of the total mechanical energy results in the Navier Stokes equations.

$$
\begin{aligned}
\frac{d}{d t} E_{T}(t)=\iiint_{\delta V_{o}} \frac{d}{d t} B(t, \vec{X}(t), \vec{u}(t, \vec{X}(t))) \rho_{o} \frac{\partial\left(X_{1}, X_{2}, X_{3}\right)}{\partial\left(R_{1}, R_{2}, R_{3}\right)} d V_{o} \\
\quad=\iiint_{\delta V_{o}} u_{i}\left(\frac{\partial u_{i}}{\partial t}+u_{j} \frac{\partial u_{i}}{\partial X_{j}}+\frac{\partial \phi}{\partial X_{i}}+\frac{1}{\rho_{o}} \frac{\partial p}{\partial X_{i}}-\frac{1}{\rho_{o}} \frac{\partial \tau_{i, j}}{\partial X_{j}}\right) \rho_{o} \frac{\partial\left(X_{1}, X_{2}, X_{3}\right)}{\partial\left(R_{1}, R_{2}, R_{3}\right)} d V_{o}=0
\end{aligned}
$$

Taking the limit of the control volume to zero,

$$
\begin{aligned}
\lim _{\delta V_{o} \rightarrow 0} \frac{1}{\delta V_{o}} \iiint_{\delta V_{o}} & \left(\frac{d}{d t} B(t, \vec{X}(t), \vec{u}(t, \vec{X}(t)))\right. \\
& \left.-\left(\frac{\partial u_{i}}{\partial t}+u_{j} \frac{\partial u_{i}}{\partial X_{j}}+\frac{\partial \phi}{\partial X_{i}}+\frac{1}{\rho_{o}} \frac{\partial p}{\partial X_{i}}-\frac{1}{\rho_{o}} \frac{\partial \tau_{i, j}}{\partial X_{j}}\right) u_{i}\right) \rho_{o} \frac{\partial\left(X_{1}, X_{2}, X_{3}\right)}{\partial\left(R_{1}, R_{2}, R_{3}\right)} d V_{o}=0
\end{aligned}
$$

Since $\rho_{o} \frac{\partial\left(X_{1}, X_{2}, X_{3}\right)}{\partial\left(R_{1}, R_{2}, R_{3}\right)}>0$ the above equation yields the desired result by reference [2] footnote on page 71 for a 1-dimensional stream.

$$
\frac{d B(t, \vec{X}(t), \vec{u}(t, \vec{X}(t)))}{d t}-\left(\frac{\partial u_{i}}{\partial t}+u_{j} \frac{\partial u_{i}}{\partial X_{j}}+\frac{\partial \phi}{\partial X_{i}}+\frac{1}{\rho_{o}} \frac{\partial p}{\partial X_{i}}-\frac{1}{\rho_{o}} \frac{\partial \tau_{i, j}}{\partial X_{j}}\right) u_{i}=0
$$

The definition of the field derivative by Theorem 1 is an additional result of this manuscript used to explain the integration result (below) being demonstrated in Section 3.3 by the Induction method and the Conclusion of this manuscript. Since $\frac{d B(t, \vec{X}(t), \vec{u}(t, \vec{X}(t)))}{d t}=\frac{D B(\vec{X}(t), t)}{D t}$, this implies the limit results in Appendix A are the equivalent to this section. In Section 3.3 the Lagrangian coordinates are identical to the Eulerian coordinates at sampled times and space by construction, although the notation differs. Thus, Section 3.3 will prove the integration result below.

$$
\begin{aligned}
\int_{0}^{t}\left(\frac{\partial u_{i}}{\partial s}+u_{j} \frac{\partial u_{i}}{\partial X_{j}}\right. & \left.+\frac{\partial \phi}{\partial X_{i}}+\frac{1}{\rho_{o}} \frac{\partial p}{\partial X_{i}}-\frac{1}{\rho_{o}} \frac{\partial \tau_{i, j}}{\partial X_{j}}\right) u_{i} d s=\int_{0}^{t} \frac{d}{d s} B(s, \vec{X}(s), \vec{u}(s, \vec{X}(s))) d s=\left.\Delta B\right|_{0} ^{t} \\
& =0
\end{aligned}
$$

\subsection{Sampling the flow field of a 1-dimensional stream and proof by induction}

In this section, the results of Section 3.1 and Appendix A will be used, therefore the same notation is used in this section. What follows is a thought experiment, where the fluid parcels in a single one-dimensional fluid stream are sampled to obtain the $k^{\text {th }}$ spatial Cartesian coordinate sample, $\vec{R}_{k}$, at sample time, $t_{k}$, using the same fixed Cartesian 3dimensional coordinate system and origin (see Figure 1) and using the same start clock time, $\mathrm{t}_{\mathrm{o}}=0$. A more sophisticated sampling experiment has been performed in a computer simulation in reference [3] for Lagrangian and Eulerian coordinates for the same fluid parcels in a stream flow field. 


\section{Javier Rivera}

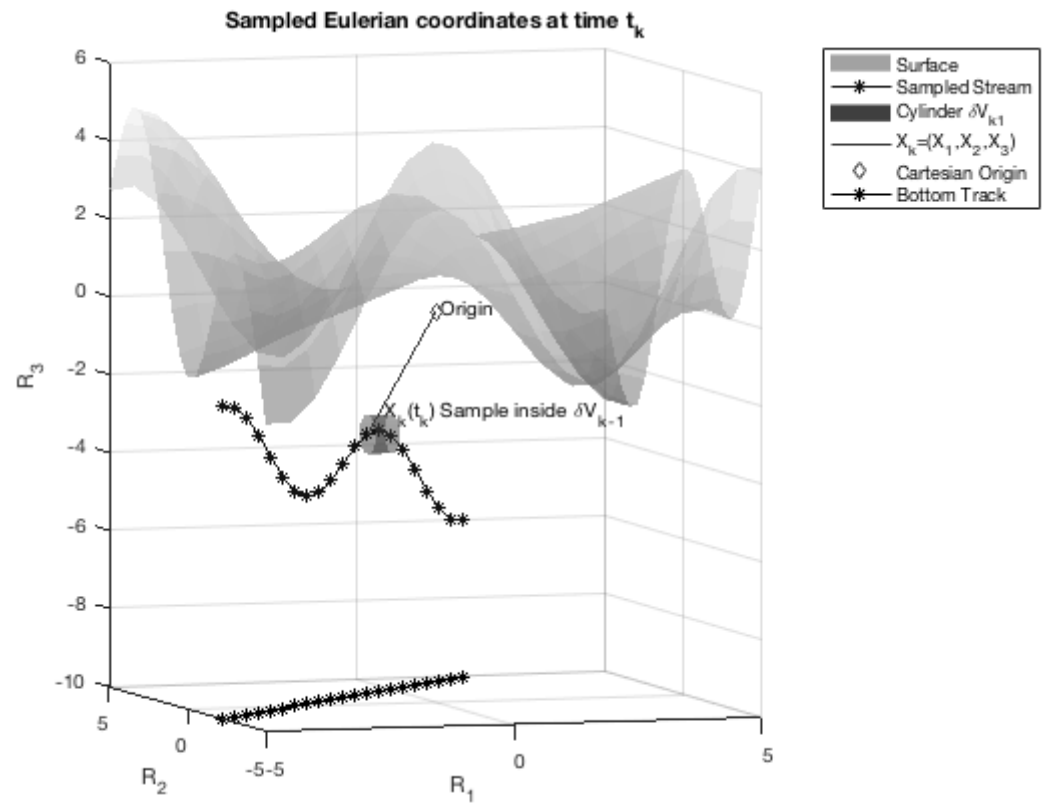

Figure 1: Spatial Sampling

The Eulerian coordinates, $\vec{X}_{k}(t)$, are sampled spatially for some fixed values Cartesian coordinate, $\vec{R}_{k}$, and sampled at the time, $t_{k}$, and the fluid parcel Lagrangian coordinates, $\vec{x}\left(\vec{R}_{k}, t_{k}\right)$, describes fluid parcels along a one-dimensional current flow that are measured with respect to the same Cartesian coordinate system and origin as the Eulerian coordinates, $\vec{X}_{k}\left(t_{k}\right)=\vec{R}_{k}$. Therefore, the sampled Lagrangian coordinates $\vec{x}\left(\vec{R}_{k}, t_{k}\right)$ and $\vec{x}\left(\vec{R}_{k-1}, t_{k-1}\right)$ may not be the same fluid parcel (this definition is not the standard Lagrangian definition because it does not use the initial position at the initial time, 0 , as the marker of a fluid parcel instead uses sampled positions and sampled times of the fluid current, unless considering the initial sample). This sampling allows unique Eulerian coordinates to be assigned to the travelling Lagrangian coordinates fluid parcels based on the same fixed valued Cartesian coordinate system for each sample or instance time $t_{k}, \vec{x}\left(\vec{R}_{k}, t_{k}\right)=\vec{R}_{k}$, with the same vector value as the sampled Eulerian coordinates, $\vec{X}_{k}=\vec{R}_{k}$. The Lagrangian coordinates of the fluid stream is sampled for a given fixed travel distance $\mathrm{L}$, and some fixed travel time $\mathrm{t}$ in the same Cartesian coordinates as the Eulerian coordinates of the fluid stream; thus, for every fluid parcel in the stream, there exists a Bernoulli line integral and, by construction, the values of the Lagrangian fluid parcel coordinates and Eulerian coordinates are the same within the 1-d stream; therefore, their velocities, $\vec{V}$, (see reference [3] equation 2 in reference) will be identical at the same sampled spatial distances and sampled times.

$$
\left.\vec{u}\left(\vec{X}_{k}, t_{k}\right)\right|_{\vec{X}_{k}=\vec{R}_{k}}=\left.\vec{V}_{\text {Euler }}\left(\vec{R}_{k}, t_{k}\right)\right|_{\vec{R}_{k}=\vec{x}\left(\vec{R}_{k}, t_{k}\right)}=\vec{V}_{\text {Lagrangian }}\left(\vec{x}\left(\vec{R}_{k}, t_{k}\right), t_{k}\right)=\vec{u}\left(\vec{x}\left(\vec{R}_{k}, t_{k}\right), t_{k}\right)
$$

Thus, the fact that the sampled Lagrangian coordinates can be substituted into the Eulerian Bernoulli equation in which the velocity field is implied but not shown since it is too clumsy of a notation. This demonstrates the material derivative [6] defined in 


\section{On the Field Derivatives and its Application to Fluids}

Appendix A, Section 3.1 and the field derivative defined in Section 2 and demonstrated in Section 3.2 are equivalent but differ in mathematical development since limit definitions are different. The sampled Bernoulli equations can be spatially integrated any time, there is a discussion involving the re-applications of (Eqs. $7 A B C)$, even if the spatial integrals are not shown. The use of the theorem of induction [4] with a time interval $\epsilon_{k} \geq 0$ $\left(\epsilon_{k}=t_{k}-t_{k-1} \ll 1\right)$ and Eulerian spatial intervals $\left|\Delta \vec{R}_{k}\right| \geq 0\left(\left|\Delta \vec{R}_{k}\right|=\left|\vec{R}_{k}-\vec{R}_{k-1}\right| \ll\right.$ 1) sampling scheme defines coordinates, $\vec{x}\left(\vec{R}_{k}, t_{k}\right)$, at times, $t_{k}$, to be used in the partial sums of the sampled Bernoulli equations. As the spatial and time sample numbers increases, the number of Lagrangian coordinates increases as well as the number of joint Eulerian control volumes increases, while keeping spatial sample $(n+1) \bar{R}=$ $\sum_{k=1}^{n+1}\left|\Delta \vec{R}_{k}\right| \leq(n+1) \delta L=L$ to a fixed distance as $\bar{R} \rightarrow 0, n \rightarrow \infty$ and time sample $t_{n+1}=$ $\sum_{k=1}^{n+1}\left(\epsilon_{k}\right)=(n+1) \bar{\epsilon}=t$ to a fixed time as $\bar{\epsilon} \rightarrow 0$ and $n \rightarrow \infty$. The total mechanical energy of the fluid is a scalar quantity, which is a constant and therefore is invariant with respect to the same Cartesian coordinate system being used.

$$
B\left(\vec{x}\left(\vec{R}_{k}, t_{k}\right), t_{k}\right)=\frac{1}{2} u_{i}^{2}\left(\vec{x}\left(\vec{R}_{k}, t_{k}\right), t_{k}\right)+\phi\left(\vec{x}\left(\vec{R}_{k}, t_{k}\right)\right)+\frac{p\left(\vec{x}\left(\vec{R}_{k}, t_{k}\right)\right)}{\rho_{o}}+\frac{1}{\rho_{o}} \int_{0}^{t_{k}} \tau_{i, j} \frac{\partial u_{i}}{\partial x_{j}} d s+I_{o}=B_{o}
$$

$$
\text { Lagrangian, } \frac{1}{2} u_{i}^{2}\left(\vec{x}_{o}, 0\right)+\phi\left(\vec{x}_{o}\right)+\frac{p\left(\vec{x}_{o}\right)}{\rho_{o}}+I_{o}=B_{o}
$$

Another explanation for (Eqs. 7DE) can be developed by applying (Eqs. 7A) to these joint Eulerian control volumes used in spatial integration as the control volume shrinks or tends to zero; therefore, the Eulerian control volumes eventually become the Lagrangian coordinates of the same or equivalent fluid parcels or particles that follow a flow defined by the Navier Stokes equations (Eq. 6) and the continuity of the fluid. The Lagrangian coordinate notation, $\vec{x}\left(\vec{R}_{k}, t_{k}\right)$, is cumbersome; thus, instead of using $\vec{x}\left(\vec{R}_{k}, t_{k}\right)$ throughout this manuscript, an equivalent compact form of the following Lagrangian coordinate notation as $\vec{x}\left(t_{k}\right)=\vec{R}_{k}$ is used, where $\mathrm{k}$ is a nonnegative integer (see footnote ${ }^{3}$ ).

Consider a small fluid cylindrical parcel with control volume, $\delta V_{o}\left(=\delta L \pi \delta r^{2}\right)$, with the small length, $\delta L$, aligned with the 1-dimensional stream flow axis at the $1^{\text {st }}$ sample. The fluid cell with Lagrangian initial coordinates, $\vec{x}(0)=\vec{x}_{o}$, is at the start of the stream cylindrical control volume circular face. The Lagrangian initial coordinates are equal in value to the Eulerian coordinates, $\vec{X}_{o}\left(=\vec{R}_{o}\right)$, and not near the physical boundaries (i.e., the fluid parcel is not stagnant, and thus the fluid parcel speed is not zero) within a much larger physical volume $V_{o}$ and have the stream control volume $\delta V_{o}$ cylinder exterior unit

\footnotetext{
${ }^{3}$ Time and spatial sampling of the fluid parcel's Lagrangian coordinates at the fixed coordinates, $\vec{R}_{k}$, along the stream's path for time $\mathrm{t}_{\mathrm{k}}$ can be expressed in terms of Lagrangian coordinates at coordinates $\vec{R}_{k-1}$ and for time $\mathrm{t}_{\mathrm{k}-1}$ via Taylor series; thus, let sampling cases $\mathrm{k}$ go to infinity as the sampling time interval $\epsilon_{k}=\left(t_{k}-t_{k-1}\right) \rightarrow 0$, and Eulerian sampling distance $\left|\Delta \vec{R}_{k}\right| \rightarrow 0$, as the sampling distance $\delta L \rightarrow 0$. (This infinitesimal sampling is not required for computational use due to Shannon's sampling theorem, as long as the simulated fluid velocity is band limited).

$\vec{x}\left(\vec{R}_{k}, t_{k}\right)=\vec{x}\left(\vec{R}_{k-1}, t_{k-1}\right)+\epsilon_{k} \partial \vec{x}\left(\vec{R}_{k-1}, t_{k-1}\right) / \partial t_{k-1}+\Delta \vec{R}_{k} \cdot \nabla \vec{x}\left(\vec{R}_{k-1}, t_{k-1}\right)+O\left(\epsilon_{k}{ }^{2},\left|\Delta \vec{R}_{k}\right|^{2}\right)$ where $\left|\Delta \vec{R}_{k}\right|=\left|\vec{R}_{k}-\vec{R}_{k-1}\right|<\delta L \ll 1, \epsilon_{k}=t_{k}-t_{k-1} \ll 1$ and the gradient $\nabla$ is with respect to $\vec{R}_{k-1}$.
} 


\section{Javier Rivera}

normal $\vec{n}_{L}$, with small radius, $\delta r<1$, i.e., $\delta V_{0}=\delta L \pi \delta r^{2} \rightarrow 0$ as $\delta r \rightarrow 0$. The Jacobian is not shown since its constant about unity for small times, $1>\Delta t=t_{1}-t_{o}=t_{1}$

$$
\begin{array}{r}
\text { limit }_{\delta r \rightarrow 0} \frac{1}{\rho_{o} \delta L \pi \delta r^{2}} \iiint_{\delta V_{0}}\left(\frac{1}{2} u_{i}^{2}\left(\vec{x}\left(t_{1}\right), t_{1}\right)+\phi\left(\overrightarrow{\vec{x}}\left(t_{1}\right)\right)+\frac{p\left(\overrightarrow{\vec{x}}\left(t_{1}\right)\right)}{\rho_{o}}+\frac{1}{\rho_{o}} \int_{0}^{t_{1}} \tau_{i, j} \frac{\partial u_{i}}{\partial x_{j}} d s+I_{o}-\right. \\
\left.B_{o}\right) \rho_{o} d V=\operatorname{limit}_{\delta r \rightarrow 0} \frac{1}{\rho_{o} \delta L \pi \delta r^{2}}\left(\frac{1}{2} u_{i}^{2}\left(\vec{x}\left(t_{1}\right), t_{1}\right)+\phi\left(\overrightarrow{\vec{x}}\left(t_{1}\right)\right)+\frac{p\left(\overrightarrow{\vec{x}}\left(t_{1}\right)\right)}{\rho_{o}}+\frac{1}{\rho_{o}} \int_{0}^{t_{1}} \tau_{i, j} \frac{\partial u_{i}}{\partial x_{j}} d s+I_{o}-\right. \\
\left.B_{o}\right) \rho_{o} \delta L \pi \delta r^{2}=\left(\frac{1}{2} u_{i}^{2}\left(\vec{x}\left(t_{1}\right), t_{1}\right)+\phi\left(\overrightarrow{\vec{x}}\left(t_{1}\right)\right)+\frac{p\left(\overrightarrow{\left.\vec{x}\left(t_{1}\right)\right)}\right.}{\rho_{o}}+\frac{1}{\rho_{o}} \int_{0}^{t_{1}} \tau_{i, j} \frac{\partial u_{i}}{\partial X_{j}} d s+I_{o}-B_{o}\right)=0
\end{array}
$$

by Mean Value Theorem of Integrals for some Lagrangian coordinate $\vec{x}\left(t_{1}\right)$ within $\delta V_{0}$ (i.e. Re-applying (Eqs. 7ABCD) with the integrand evaluated at some sampled coordinates $\vec{R}_{1}$ (within $\vec{x}_{o}^{\prime}$ s or $\vec{R}_{o}^{\prime}$ s cylinder control volume)). Defining a fluid parcel Lagrangian coordinates as $\vec{x}\left(t_{1}\right)=\vec{R}_{1}$ (equivalent Eulerian coordinates at time $t_{l}$ ) results in the sampled Bernoulli equation 1 (Eq. 8).

$$
B\left(\vec{x}\left(t_{1}\right), t_{1}\right)=\frac{1}{2} u_{i}^{2}+\phi\left(\vec{x}\left(t_{1}\right)\right)+\frac{p\left(\vec{x}\left(t_{1}\right)\right)}{\rho_{o}}+\frac{1}{\rho_{o}} \int_{0}^{t_{1}} \tau_{i, j} \frac{\partial u_{i}}{\partial x_{j}} d s+I_{o}=B_{o}
$$

For the sample $\mathrm{k}$ fluid parcel along the 1-dimensional stream (see Figure 1), where the sample time $t_{k}$ is the fluid cell's Lagrangian coordinates, $\vec{x}\left(t_{k}\right)$, and the fluid parcel is within the relatively small neighborhood of $\delta V_{k-1},(E q .9)$, if the fluid's maximum speed (parallel to the cylinder's $\delta L$ axis exterior unit normal) is denoted by $U_{k, L}=\operatorname{Max} \vec{u} \cdot \vec{n}_{L}$ > 0 , then the time $t_{k}$ required to be within the time interval is $\left[(k-1) \delta L / U_{k-1, r}, k \delta L /\right.$ $\left.U_{k, r}\right]$; otherwise, the $\vec{x}\left(t_{k}\right)$ fluid parcel location is outside the axial spatial integration of the cylindrical control volume $\delta V_{k-1}$ since control volume encloses the stream only entry and exit is through the cylinder circular faces.

By re-applying (Eqs. $7 D E F)$ with the integrand evaluated at some Eulerian coordinates $\vec{R}_{k}$ (within $\vec{R}_{k-1}^{\prime}$ s control volume), for a fluid parcel Lagrangian coordinates, $\vec{x}\left(t_{k}\right)=\vec{R}_{k}$, results in the sampled Bernoulli equation $k$ are subtracted by Bernoulli equation $k-1$. Subtract the $k-1$ sampled Bernoulli equation $B\left(\vec{x}\left(t_{k-1}\right), t_{k-1}\right)$ from the $k^{\text {th }}$ sampled Bernoulli equations $B\left(\vec{x}\left(t_{k}\right), t_{k}\right)$, and for simplicity, let $V(\vec{x}(t))=\frac{p(\vec{x}(t))}{\rho_{o}}+\phi(\vec{x}(t))$ (if $k=1$ use $(E q .7 E)$ ) to obtain the difference sample Bernoulli equations for each difference $k$ in (Eq. 9.)

$$
\begin{aligned}
B\left(\vec{x}\left(t_{k}\right), t_{k}\right)-B & \left(\vec{x}\left(t_{k-1}\right), t_{k-1}\right) \\
& =\frac{1}{2} u_{i}^{2}\left(\vec{x}\left(t_{k}\right), t_{k}\right)-\frac{1}{2} u_{i}^{2}\left(\vec{x}\left(t_{k-1}\right), t_{k-1}\right)+v\left(\vec{x}\left(t_{k}\right)\right)-v\left(\vec{x}\left(t_{k-1}\right)\right)+\frac{1}{\rho_{o}} \int_{t_{k-1}}^{t_{k}} \tau_{i j} \frac{\partial u_{i}}{\partial x_{j}} d s
\end{aligned}
$$

The implication is that the difference of mechanical energy per unit mass in (Eq. 9) is a constant zero within the fluid 1-dimensional stream, and therefore, the cylindrical control volume moves to a new start position, $\vec{x}\left(t_{k}\right)$ (i.e. by placing the start of control volume's face center at $\left.\vec{x}\left(t_{k}\right)\right)$. The sample times in the equations can be defined as $t_{k}=t_{k-1}+\epsilon_{k}$, for integer $k \geq 1$; when $k=1$, define $t_{0}=0$, and $t_{1}=\epsilon_{1} \geq 0$. For each of the cylindrical volumes, $\delta V_{k}=\delta L \pi \delta r^{2}$, are equal to the initial volume, $\delta V_{o}$. Note that the fluid parcel is assumed to be mostly advective transport. For each sampled Bernoulli formula, $k$, there is a Eulerian coordinate $\vec{X}_{k}$ that is equal to the Lagrangian coordinates $\vec{x}\left(t_{k}\right)$, within the cylinder's volume of Lagrangian coordinate $\vec{x}\left(t_{k-1}\right)$ or $\vec{X}_{k-1}$ by construction. 
On the Field Derivatives and its Application to Fluids

Set $k$ equal to 2 and start this argument over again for sample equation $k$ equal to 3 , and so on, when we arrive to $k+1$ difference equations; thus, the $k+1$ cylinder start face center is located at $\vec{x}\left(t_{k}\right)$. As the number of difference cases, $k$, increases with the condition that the cylinder's volume decreases (i.e., $\delta L \rightarrow 0, \delta r \rightarrow 0)$ and re-applying $(E q .7 F$ with Lagrangian coordinates) for each shrinking control volume as $k$ increases, this process increases the resolution. Therefore, the Lagrangian coordinates of the fluid parcel will match the Eulerian coordinate samples of the same fluid parcel by construction because the fluid flow satisfies the Navier Stokes equations and the equation of continuity. See Section 3.1 for proof for the case $k=1$ and this result can be repeated for all $k$ 's. See Section 3.2 for a proof of the integration of the field derivative used in this section. Unfortunately, due to space concerns the notation is the same for Section 3.2 but different notation found in Section 3.2, $B\left(\vec{x}\left(t_{k}\right), t_{k}\right)=B\left(t_{k}, \vec{x}\left(t_{k}\right), \vec{u}\left(\vec{x}\left(t_{k}\right), t_{k}\right)\right.$ i.e. fluid velocity field is implied in the argument of the Bernoulli function but not shown in Section 3.3, but matches notation used in Section 3.1.

Difference $k=1$, holds for some $t_{1} \in\left[0, \frac{\delta L}{U_{1, L}}\right]$ and for coordinates $\vec{x}\left(t_{1}\right)$ and $\vec{x}(0) \in \delta V_{0}$

$$
\begin{gathered}
B\left(\vec{x}\left(t_{1}\right), t_{1}\right)-B(\vec{x}(0), 0)=\frac{1}{2} u_{i}^{2}\left(\vec{x}\left(t_{1}\right), t_{1}\right)-\frac{1}{2} u_{i}^{2}(\vec{x}(0), 0)+V\left(\vec{x}\left(t_{1}\right)\right)-V(\vec{x}(0))+\frac{1}{\rho_{o}} \int_{0}^{t_{1}} \tau_{i, j} \frac{\partial u_{i}}{\partial x_{j}} d s \\
=0
\end{gathered}
$$

Difference $k=2$, holds for some $t_{2} \in\left[\frac{\delta L}{U_{1, L}}, \frac{2 \delta L}{U_{2, L}}\right]$ and for coordinates $\vec{x}\left(t_{2}\right)$ and $\vec{x}\left(t_{1}\right) \in \delta V_{1}$

$$
\begin{gathered}
B\left(\vec{x}\left(t_{2}\right), t_{2}\right)-B\left(\vec{x}\left(t_{1}\right), t_{1}\right)=\frac{1}{2} u_{i}^{2}\left(\vec{x}\left(t_{2}\right), t_{2}\right)-\frac{1}{2} u_{i}^{2}\left(\vec{x}\left(t_{1}\right), t_{1}\right)+V\left(\vec{x}\left(t_{2}\right)\right)-V\left(\vec{x}\left(t_{1}\right)\right)+\frac{1}{\rho_{o}} \int_{t_{1}}^{t_{2}} \tau_{i, j} \frac{\partial u_{i}}{\partial x_{j}} d s \\
=0
\end{gathered}
$$

Difference $k=\mathrm{n}$, holds for some $t_{n} \in\left[\frac{(n-1) \delta L}{U_{n-1, L}}, \frac{n \delta L}{U_{n, L}}\right]$ and for coordinates

$$
\begin{aligned}
& \vec{x}\left(t_{n}\right) \text { and } \vec{x}\left(t_{n-1}\right) \in \delta V_{n-1} \\
& B\left(\vec{x}\left(t_{n}\right), t_{n}\right)-B\left(\vec{x}\left(t_{n-1}\right), t_{n-1}\right)=\frac{1}{2} u_{i}^{2}\left(\vec{x}\left(t_{n}\right), t_{n}\right)-\frac{1}{2} u_{i}^{2}\left(\vec{x}\left(t_{n-1}\right), t_{n-1}\right)+V\left(\vec{x}\left(t_{n}\right)\right)-V\left(\vec{x}\left(t_{n-1}\right)\right)+\frac{1}{\rho_{o}} \int_{t_{n-1}}^{t_{n}} \tau_{i, j} \frac{\partial u_{i}}{\partial x_{j}} d s \\
& \quad=0
\end{aligned}
$$

Difference $k=\mathrm{n}+1$, holds for some $t_{n+1} \in\left[\frac{n \delta L}{U_{n, L}}, \frac{(n+1) \delta L}{U_{(n+1), L}}\right]$ and for coordinates

$$
\begin{aligned}
& \vec{x}\left(t_{n+1}\right) \text { and } \vec{x}\left(t_{n}\right) \in \delta V_{n} \\
& B\left(\vec{x}\left(t_{n+1}\right), t_{n+1}\right)-B\left(\vec{x}\left(t_{n}\right), t_{n}\right)=\frac{1}{2} u_{i}^{2}\left(\vec{x}\left(t_{n+1}\right), t_{n+1}\right)-\frac{1}{2} u_{i}^{2}\left(\vec{x}\left(t_{n}\right), t_{n}\right)+V\left(\vec{x}\left(t_{n+1}\right)\right)-V\left(\vec{x}\left(t_{n}\right)\right)+\frac{1}{\rho_{o}} \int_{t_{n}}^{t_{n+1}} \tau_{i, j} \frac{\partial u_{i}}{\partial x_{j}} d s \\
& =0
\end{aligned}
$$

Add each difference of the sampled Bernoulli equation into a partial sum composed of samples $k$ equal to 1 and 2, and so on. Applying the induction method, for a case $\mathrm{n}$ equal to 1 , the sum of equations for differences $k$ equal to 1 and 2 holds by inspection for $s \in\left[0, \frac{2 \delta L}{U_{2, L}}\right]$.

\section{Induction case 1 holds by inspection,}

$$
\sum_{k=1}^{2}\left(B\left(\vec{x}\left(t_{k}\right), t_{k}\right)-B\left(\vec{x}\left(t_{k-1}\right), t_{k-1}\right)\right)=B\left(\vec{x}\left(t_{2}\right), t_{2}\right)-B(\vec{x}(0), 0)=\int_{0}^{t_{2}} d B(\vec{x}(s), s)
$$




\section{Javier Rivera}

$$
=\frac{1}{2} u_{i}^{2}\left(\vec{x}\left(t_{2}\right), t_{2}\right)-\frac{1}{2} u_{i}^{2}(\vec{x}(0), 0)+V\left(\vec{x}\left(t_{2}\right)\right)-V(\vec{x}(0))+\frac{1}{\rho_{o}} \int_{0}^{t_{2}} \tau_{i, j} \frac{\partial u_{i}}{\partial x_{j}} d s=0
$$

\section{Induction case $\mathbf{n}+\mathbf{1}$,}

Assume case $n$ holds, to show the general case $n+1$ holds, add the finite sum of differences from 1 to $n+1$; the partial sums will increase by induction. The partial sum is valid for the union of the time intervals. The time integral holds for $s \in\left[0, \frac{(n+1) \delta L}{U_{(n+1), L}}\right]$. The case $\mathrm{n}+1$ is given by the finite sum below, which also holds by inspection and using the fundamental theorem of calculus [5]. By definition, $t_{n+1}=\sum_{k=1}^{n+1}\left(\epsilon_{k}\right)=(n+1) \bar{\epsilon}=t$.

\section{Case Bernoulli Integral n holds by assumption,}

$$
\sum_{k=1}^{n}\left(B\left(\vec{x}\left(t_{k}\right), t_{k}\right)-B\left(\vec{x}\left(t_{k-1}\right), t_{k-1}\right)\right)=\left(B\left(\vec{x}\left(t_{n}\right), t_{n}\right)-B(\vec{x}(0), 0)\right)=\int_{0}^{t_{n}} d B(\vec{x}(s), s)
$$

\section{Case Bernoulli integral $\mathbf{n}+1$,}

$$
\begin{aligned}
& \operatorname{Int}_{n+1}=\sum_{k=1}^{n+1}\left(\left(B\left(\vec{x}\left(t_{k}\right), t_{k}\right)-B\left(\vec{x}\left(t_{k-1}\right), t_{k-1}\right)\right)\right)=\left(B\left(\vec{x}\left(t_{n+1}\right), t_{n+1}\right)-B\left(\vec{x}\left(t_{n}\right), t_{n}\right)\right) \\
& +\sum_{k=1}^{n}\left(B\left(\vec{x}\left(t_{k}\right), t_{k}\right)-B\left(\vec{x}\left(t_{k-1}\right), t_{k-1}\right)\right) \\
& \operatorname{Int}_{n+1}=\left(B\left(\vec{x}\left(t_{n+1}\right), t_{n+1}\right)-B\left(\vec{x}\left(t_{n}\right), t_{n}\right)\right)+\left(B\left(\vec{x}\left(t_{n}\right), t_{n}\right)-B(\vec{x}(0), 0)\right) \\
& \operatorname{Int}_{n+1}=\int_{t_{n}}^{t_{n+1}} d B(\vec{x}(s), s)+\int_{0}^{t_{n}} d B(\vec{x}(s), s)
\end{aligned}
$$

Therefore,

$$
\operatorname{Int}_{n+1}=\left(B\left(\vec{x}\left(t_{n+1}\right), t_{n+1}\right)-B(\vec{x}(0), 0)\right)=\int_{0}^{t_{n+1}} d B(\vec{x}(s), s)
$$

where the time integral of a field derivative results in the difference evaluated at the endpoints of the time integral due to the fundamental theorem of calculus. Moreover, the finite sum above and below holds by inspection due to the term-by-term cancellation.

\section{Case n Bernoulli differences sum holds by inspection,}

$$
\begin{aligned}
\sum_{k=1}^{n} \frac{1}{2} u_{i}^{2}\left(\vec{x}\left(t_{k}\right), t_{k}\right) & -\frac{1}{2} u_{i}^{2}\left(\vec{x}\left(t_{k-1}\right), t_{k-1}\right)+V\left(\vec{x}\left(t_{k}\right)\right)-V\left(\vec{x}\left(t_{k-1}\right)\right)+\frac{1}{\rho_{o}} \int_{t_{k-1}}^{t_{k}} \tau_{i, j} \frac{\partial u_{i}}{\partial x_{j}} d s \\
= & \frac{1}{2} u_{i}^{2}\left(\vec{x}\left(t_{n}\right), t_{n}\right)-\frac{1}{2} u_{i}^{2}(\vec{x}(0), 0)+V\left(\vec{x}\left(t_{n}\right)\right)-V(\vec{x}(0))+\frac{1}{\rho_{o}} \int_{0}^{t_{n}} \tau_{i, j} \frac{\partial u_{i}}{\partial x_{j}} d s
\end{aligned}
$$


On the Field Derivatives and its Application to Fluids

\section{Case $n+1$ Bernoulli differences sum,}

$$
\begin{gathered}
\text { Bernoulli } \Delta \text { Sum }=\sum_{k=1}^{n+1} \frac{1}{2} u_{i}^{2}\left(\vec{x}\left(t_{k}\right), t_{k}\right)-\frac{1}{2} u_{i}^{2}\left(\vec{x}\left(t_{k-1}\right), t_{k-1}\right)+V\left(\vec{x}\left(t_{k}\right)\right)-V\left(\vec{x}\left(t_{k-1}\right)\right)+\frac{1}{\rho_{o}} \int_{t_{k-1}}^{t_{k}} \tau_{i, j} \frac{\partial u_{i}}{\partial x_{j}} d s \\
=\left[\frac{1}{2} u_{i}^{2}\left(\vec{x}\left(t_{n+1}\right), t_{n+1}\right)-\frac{1}{2} u_{i}^{2}\left(\vec{x}\left(t_{n}\right), t_{n}\right)+V\left(\vec{x}\left(t_{n+1}\right)\right)-V\left(\vec{x}\left(t_{n}\right)\right)+\frac{1}{\rho_{o}} \int_{t_{n}}^{t_{n+1}} \tau_{i, j} \frac{\partial u_{i}}{\partial x_{j}} d s\right. \\
\left.+\sum_{k=1}^{n} \frac{1}{2} u_{i}^{2}\left(\vec{x}\left(t_{k}\right), t_{k}\right)-\frac{1}{2} u_{i}^{2}\left(\vec{x}\left(t_{k-1}\right), t_{k-1}\right)+V\left(\vec{x}\left(t_{k}\right)\right)-V\left(\vec{x}\left(t_{k-1}\right)\right)+\frac{1}{\rho_{o}} \int_{t_{k-1}}^{t_{k}} \tau_{i, j} \frac{\partial u_{i}}{\partial x_{j}} d s\right] \\
\text { Bernoulli } \Delta S \text { Sum }=\left[\frac{1}{2} u_{i}^{2}\left(\vec{x}\left(t_{n+1}\right), t_{n+1}\right)-\frac{1}{2} u_{i}^{2}\left(\vec{x}\left(t_{n}\right), t_{n}\right)+V\left(\vec{x}\left(t_{n+1}\right)\right)-V\left(\vec{x}\left(t_{n}\right)\right)+\frac{1}{\rho_{o}} \int_{t_{n}}^{t_{n+1}} \tau_{i, j} \frac{\partial u_{i}}{\partial x_{j}} d s\right. \\
\left.+\frac{1}{2} u_{i}^{2}\left(\vec{x}\left(t_{n}\right), t_{n}\right)-\frac{1}{2} u_{i}^{2}(\vec{x}(0), 0)+V\left(\vec{x}\left(t_{n}\right)\right)-V(\vec{x}(0))+\frac{1}{\rho_{o}} \int_{0}^{t_{n}} \tau_{i, j} \frac{\partial u_{i}}{\partial x_{j}} d s\right]
\end{gathered}
$$

The combined induction case $\mathrm{n}+1$ result is shown below.

$\int_{0}^{t_{n+1}} d B(\vec{x}(s), s)=\frac{1}{2} u_{i}^{2}\left(\vec{x}\left(t_{n+1}\right), t_{n+1}\right)-\frac{1}{2} u_{i}^{2}(\vec{x}(0), 0)+V\left(\vec{x}\left(t_{n+1}\right)\right)-V(\vec{x}(0))+\frac{1}{\rho_{o}} \int_{0}^{t_{n+1}} \tau_{i, j} \frac{\partial u_{i}}{\partial x_{j}} d s=0$

By induction and repeated use of $(E q .7 F)$, as $n \rightarrow \infty$, with $\delta L \rightarrow 0, \delta r \rightarrow 0$ and as $t_{k}-t_{k-1}=\epsilon_{k} \rightarrow 0$, as $\bar{\epsilon} \rightarrow 0, U_{(n+1), L} \rightarrow U_{L},(n+1) \delta L \rightarrow L$; thus, the limit $_{n \rightarrow \infty} t_{n+1}=$ limit $_{n \rightarrow \infty}(n+1) \bar{\epsilon}=t$ converges by construction. Therefore,

$$
\begin{aligned}
\int_{0}^{\text {limit }_{n \rightarrow \infty} t_{n+1}} \frac{d B(\vec{x}(s), s)}{d s} d s & =\text { limit }_{n \rightarrow \infty}\left(\frac{1}{2} u_{i}^{2}\left(\vec{x}\left(t_{n+1}\right), t_{n+1}\right)-\frac{1}{2} u_{i}^{2}(\vec{x}(0), 0)+V\left(\vec{x}\left(t_{n+1}\right)\right)-V(\vec{x}(0))\right) \\
& +\frac{1}{\rho_{o}} \int_{0}^{l i m i t}{ }_{n \rightarrow \infty} t_{n+1} \tau_{i, j} \frac{\partial u_{i}}{\partial x_{j}} d s=0
\end{aligned}
$$

The previous equations hold true because the sum is performed before the limits were evaluated. The limits exist and converge uniformly since the fluid velocity and the potential functions are continuous functions of time and space, and since limit $_{n \rightarrow \infty} t_{n+1}=$ t converges uniformly by construction. By adding and subtracting the initial constant internal energy, all the cases of the partial sums hold true for the union of the time intervals, $t \in\left[0, \frac{L}{U_{L}}\right]$. The final equation yields a line integral of the material derivative of the Bernoulli formula within a 1-dimensional stream. Therefore, by the law of induction and the fundamental theorem of calculus [5], the Bernoulli formula holds true, as shown below.

$$
\begin{aligned}
\int_{0}^{t} \frac{d B(\vec{x}(s), s)}{d s} d s & =\frac{1}{2} u_{i}^{2}(\vec{x}(t), t)+V(\vec{x}(t))+\frac{1}{\rho_{o}} \int_{0}^{t} \tau_{i, j} \frac{\partial u_{i}}{\partial x_{j}} d s+I_{o}-\frac{1}{2} u_{i}^{2}\left(\vec{x}_{o}, 0\right)-V\left(\vec{x}_{o}\right)-I_{o} \\
& =0
\end{aligned}
$$

where we set the time to 0 in $\vec{x}(t)$ to obtain $\vec{x}_{o}$. 


\section{Javier Rivera}

Bernoulli's principle can be stated as $\left(E q\right.$. 10) below, with $V(\vec{x}(t))$ replaced by $\frac{p(\vec{x}(t))}{\rho_{o}}+$ $\phi(\vec{x}(t))$.

$$
B(\vec{x}(t), t)=\frac{1}{2} u_{i}^{2}(\vec{x}(t), t)+\phi(\vec{x}(t))+\frac{p(\vec{x}(t))}{\rho_{o}}+\frac{1}{\rho_{o}} \int_{0}^{t} \tau_{i, j} \frac{\partial u_{i}}{\partial x_{j}} d s+I_{o}=B_{o}
$$

This equation is the same result obtained with Eulerian coordinates in $(E q .7 A B C)$, but the interpretation of a line integral is difficult to interpret since the Eulerian coordinates are fixed in space. Another alternative to $(E q .10)$ is given by the equation below,

$$
\Delta B=B(\vec{x}(t), t)-B\left(\vec{x}_{o}, 0\right)=(\Delta K+\Delta V+\Delta I)=0
$$

and

$$
\begin{gathered}
\Delta K=\frac{1}{2}\left(u_{i}^{2}(\vec{x}(t), t)-u_{i}^{2}\left(\vec{x}_{o}, 0\right)\right) \\
\left.\Delta V=\phi(\vec{x}(t))+\frac{p(\vec{x}(t))}{\rho_{o}}-\phi\left(\vec{x}_{o}\right)\right)-\frac{p\left(\vec{x}_{o}\right)}{\rho_{o}} \\
\Delta I=\frac{1}{\rho_{o}} \int_{0}^{t} \tau_{i, j}(\vec{x}(s), s) \frac{\partial u_{i}(\vec{x}(s), s)}{\partial x_{j}} d s .
\end{gathered}
$$

Substituting the fluid shear stress for incompressible fluids given by [1]

Thus,

$$
\tau_{i, j}(\vec{x}(s), s)=2 \mu e_{i, j}(\vec{x}(s), s)=\mu\left(\frac{\partial u_{i}(\vec{x}(s), s)}{\partial x_{j}}+\frac{\partial u_{j}(\vec{x}(s), s)}{\partial x_{i}}\right)
$$

$$
\begin{aligned}
\frac{1}{2}\left(u_{i}^{2}(\vec{x}(t), t)-\right. & \left.\left.u_{i}^{2}\left(\vec{x}_{o}, 0\right)\right)+\phi(\vec{x}(t))+\frac{p(\vec{x}(t))}{\rho_{o}}-\phi\left(\vec{x}_{o}\right)\right)-\frac{p\left(\vec{x}_{o}\right)}{\rho_{o}} \\
& +\frac{\mu}{\rho_{o}} \int_{0}^{t}\left(\frac{\partial u_{i}(\vec{x}(s), s)}{\partial x_{j}}+\frac{\partial u_{j}(\vec{x}(s), s)}{\partial x_{i}}\right) \frac{\partial u_{i}(\vec{x}(s), s)}{\partial x_{j}} d s=0
\end{aligned}
$$

Since $\frac{\mu}{\rho_{o}}=v$ is the kinematic viscosity, and by performing the usual dimensional analysis the Bernoulli equation to obtain the Reynolds number, $\operatorname{Re}=\frac{L U(\vec{x}(t), t)}{v}$, the fluid Reynolds number is given in (Eq. 11) below after cancelling the initial internal energy per unit mass. The Bernoulli's principle $(E q$. 11) can be restated as the first integral of the Navier Stokes equation, i.e., the time integral of the Navier Stokes yields the Bernoulli equation (recall this equation is valid within the volume integral sign and is obtained by integration by parts of the dissipation term).

Or

$$
\begin{gathered}
\Delta B=\frac{1}{2} u_{i}^{2}(\vec{x}(t), t)-\frac{1}{2} u_{i}^{2}\left(\vec{x}_{o}, 0\right)+\frac{1}{R e} \int_{0}^{t}\left(\frac{\partial u_{i}(\vec{x}(s), s)}{\partial x_{j}}+\frac{\partial u_{j}(\vec{x}(s), s)}{\partial x_{i}}\right) \frac{\partial u_{i}(\vec{x}(s), s)}{\partial x_{j}} d s \\
+\phi(\vec{x}(t))+\frac{p(\vec{x}(t))}{\rho_{o}}-\phi\left(\vec{x}_{o}\right)-\frac{p\left(\vec{x}_{o}\right)}{\rho_{o}}=0
\end{gathered}
$$


On the Field Derivatives and its Application to Fluids

$$
\begin{aligned}
\iiint_{V(t)} \text { limit }_{\Delta t \rightarrow 0} \frac{\Delta B}{\Delta t} \rho_{o} d V & =\iiint_{V_{0}} \operatorname{limit}_{\Delta t \rightarrow 0} \frac{(\Delta K+\Delta I+\Delta V)}{\Delta t} \rho_{o} \frac{\partial\left(X_{1}, X_{2}, X_{3}\right)}{\partial\left(R_{1}, R_{2}, R_{3}\right)} d V_{o} \\
& =\iiint_{V_{0}} \frac{d}{d t}(K+V+I) \rho_{o} \frac{\partial\left(X_{1}, X_{2}, X_{3}\right)}{\partial\left(R_{1}, R_{2}, R_{3}\right)} d V_{o}=\frac{d}{d t} \iiint_{V_{0}} B(\vec{x}(t), t) \rho_{o} \frac{\partial\left(X_{1}, X_{2}, X_{3}\right)}{\partial\left(R_{1}, R_{2}, R_{3}\right)} d V_{o}=\frac{d E_{T}}{d t} \\
& =0
\end{aligned}
$$

Therefore, $\frac{d}{d t}\left(H+\frac{3}{2} I\right)=\frac{d}{d t}(K+V+I)=\frac{d E_{T}}{d t}=0$ in (Eq. 4.4 in reference [1]) neglected the volume integrals, which was the original claim, and the time differentiation referred to is the material derivative or field derivative.

\section{Hamilton-Jacobi partial differential equation of incompressible fluids}

This section will develop the Hamilton Jacobi partial differential equation of incompressible fluids is based on the theorem in page 133 of Gelfand and Fomin's book Calculus of Variations [11] which was adapted to be used for fluid mechanics using the field derivative operator instead of the total derivative used in the theorem in reference [11].

Theorem 3. Hamilton Jacobi partial differential equation of incompressible fluids.

The first-order system of the Eulerian coordinates defines the fluid velocity

$$
\frac{d X_{i}(t)}{d t}=u_{i}\left(X_{i}(t), t\right)
$$

is a field for the second order system of the Eulerian coordinates given by

$$
\frac{d^{2} X_{i}}{d t^{2}}=-\frac{\partial \phi}{\partial X_{i}}-\frac{1}{\rho_{o}} \frac{\partial p}{\partial X_{i}}+v \frac{\partial^{2}}{\partial X_{j}^{2}} \frac{d X_{i}(t)}{d t}
$$

if and only if the velocity field satisfies the Hamilton-Jacobi partial differential equations of incompressible fluids

$$
\frac{d u_{i}}{d t}=\frac{\partial u_{i}}{\partial t}+u_{j} \frac{\partial u_{i}}{\partial X_{j}}=-\frac{\partial \phi}{\partial X_{i}}-\frac{1}{\rho_{o}} \frac{\partial p}{\partial X_{i}}+v \frac{\partial^{2} u_{i}}{\partial X_{j}^{2}}
$$

for the second order system of the Eulerian coordinates above.

For the complete proof see reference [11]. Consider the following optimization of the functional, $J\left[X_{i}, u_{i}\right]$, where the definition of the field derivative of the sampled fluid parcels coordinates and the fluid field velocity is used.

$$
J\left[X_{i}, u_{i}\right]=\int_{0}^{t 1} \iiint_{V_{o}} L\left(t, X_{i}, \frac{d X_{i}}{d t} u_{i}, \frac{\partial u_{i}}{\partial X_{j}}\right) \rho_{o} d V d t=\int_{0}^{t 1} \iiint_{V_{o}}\left(u_{i} \frac{d X_{i}}{d t}-H\left(t, X_{i}, u_{i}, \frac{\partial u_{i}}{\partial X_{j}}\right)\right) \rho_{o} d V d t
$$

Following reference [1] and the comments made to me by Darryl D. Holm [12].

$$
\frac{d X_{i}}{d t}-u_{i}=0
$$

Where the Hamiltonian field function was defined in [1] as

$$
H\left(t, X_{i}, u_{i}, \frac{\partial u_{i}}{\partial X_{j}}\right)=\frac{1}{2} u_{i}^{2}+\phi(\vec{X})+\frac{p(\vec{X})}{\rho_{o}}-\frac{1}{2 \rho_{o}} \int_{0}^{t} \tau_{i, j} \frac{\partial u_{i}}{\partial X_{j}} d s-\frac{1}{2} I_{o}
$$

Using the Lagrange multipliers method, introduce the constraint into the functional $J\left[X_{i}, u_{i}\right]$ so the Eulerian coordinates and Eulerian velocity can be varied independently $[1]$. 


\section{Javier Rivera}

$$
J\left[X_{i}, u_{i}\right]=\int_{0}^{t 1} \iiint_{V_{o}}\left(u_{i} \frac{d X_{i}}{d t}-H\left(t, X_{i}, u_{i}, \frac{\partial u_{i}}{\partial X_{j}}\right)+\lambda_{i}\left(\frac{d X_{i}}{d t}-u_{i}\right)\right) \rho_{o} d V d t
$$

A Taylor series approximation (not reproduced here) results in equations 2.13 and 2.15 in [1] are below in which added restrictions were added in the brackets to make clear what's being differentiated.

$\delta X_{i}:\left[\frac{\partial L}{\partial X_{i}}-\frac{d}{d t}\left(\frac{\partial L}{\partial \frac{d X_{i}}{d t}}\right)\right]_{\text {fixed } u_{i}}=-\frac{\partial H}{\partial X_{i}}-\frac{d}{d t}\left(u_{i}+\lambda_{i}\right)=0$

$\delta u_{i}:\left[\frac{\partial L}{\partial u_{i}}+\frac{\delta L}{\delta u_{i}}\right]_{\text {fixed } X_{i}}=\frac{d X_{i}}{d t}-\frac{\partial H}{\partial u_{i}}-\frac{\delta H}{\delta u_{i}}-\lambda_{i}=0$

Simplifying,

$\delta X_{i}: \frac{d u_{i}}{d t}=-\frac{\partial H}{\partial X_{i}}-\frac{d \lambda_{i}}{d t}$

$\delta u_{i}: \frac{d X_{i}}{d t}=\frac{\partial H}{\partial u_{i}}+\frac{\delta H}{\delta u_{i}}+\lambda_{i}$

The variational derivative of the Hamiltonian is with respect to the $1 / 2$ of the Internal energy integral, where there is no implied sum inside the time integral on the ratio of $i^{\text {th }}$ index virtual velocities (equations 2.17 in [1], and $t>t_{i 2}^{+} \geq 0$ ).

Hence

$$
-\frac{\delta H}{\delta u_{i}}=\frac{1}{2} \frac{\delta I}{\delta u_{i}}=-\int_{t_{i 2}^{+}}^{t}\left(\sum_{j=1}^{3} \frac{1}{\rho_{o}} \frac{\partial \tau_{i, j}}{\partial x_{j}}\right) \frac{\delta u_{i}\left(X_{i}(s), s\right)}{\delta u_{i}\left(X_{i}(t), t\right)} d s
$$

$$
\frac{d X_{i}(t)}{d t}=u_{i}\left(X_{i}(t), t\right)+\int_{t_{i 2}^{+}}^{t}\left(\sum_{j=1}^{3} \frac{1}{\rho_{o}} \frac{\partial \tau_{i, j}}{\partial x_{j}}\right) \frac{\delta u_{i}\left(X_{i}(s), s\right)}{\delta u_{i}\left(X_{i}(t), t\right)} d s+\lambda_{i}
$$

Thus, since $\frac{d X_{i}(t)}{d t}=u_{i}\left(X_{i}(t), t\right)$, therefore the Lagrange multiplier is given as,

$$
\lambda_{i}=-\int_{t_{i 2}^{+}}^{t}\left(\sum_{j=1}^{3} \frac{1}{\rho_{o}} \frac{\partial \tau_{i, j}}{\partial x_{j}}\right) \frac{\delta u_{i}\left(X_{i}(s), s\right)}{\delta u_{i}\left(X_{i}(t), t\right)} d s
$$

where there is no implied in the $i^{\text {th }}$ index sum inside the time integral. Performing the field derivative on the above equation, by epsilon-delta continuity (See reference [1] for details), and substituting into the second equation we obtain (since $\tau_{i, j}=2 \mu e_{i, j}$, see reference [1], equations 2.19-2.23)

$$
\begin{gathered}
-\frac{d}{d t} \lambda_{i}=\frac{1}{\rho_{o}} \frac{\partial \tau_{i, j}}{\partial x_{j}}=v \Delta u_{i} \\
\frac{d u_{i}}{d t}=-\frac{\partial H}{\partial X_{i}}-\frac{d \lambda_{i}}{d t}=-\frac{\partial H}{\partial X_{i}}+\sum_{j=1}^{3} \frac{1}{\rho_{o}} \frac{\partial \tau_{i, j}}{\partial x_{j}}=-\frac{\partial \phi}{\partial X_{i}}-\frac{1}{\rho_{o}} \frac{\partial p}{\partial X_{i}}+v \Delta u_{i}
\end{gathered}
$$


On the Field Derivatives and its Application to Fluids

So the Hamilton-Jacobi system of partial differential equations is

$$
\begin{gathered}
\frac{d X_{i}(t)}{d t}=u_{i}\left(X_{i}(t), t\right) \\
\frac{d u_{i}}{d t}=\frac{\partial u_{i}}{\partial t}+u_{j} \frac{\partial u_{i}}{\partial X_{j}}=-\frac{\partial \phi}{\partial X_{i}}-\frac{1}{\rho_{o}} \frac{\partial p}{\partial X_{i}}+v \Delta u_{i}
\end{gathered}
$$

See the example of field derivative of fluid velocity field in Section 2.1.

Q.E.D.

\section{Conclusion}

This manuscript has demonstrated how Bernoulli's principle for incompressible viscous fluids can be formally obtained. The Bernoulli function, $B(\vec{x}(t), t)$, is a constant within the fluid's stream control volume. The integral of the material derivative of the Bernoulli function, $B(\vec{x}(t), t)=\frac{1}{2} u_{i}^{2}(\vec{x}(t), t)+\phi(x(t))+\frac{p(\vec{x}(t))}{\rho_{o}}+\frac{1}{\rho_{o}} \int_{0}^{t} \tau_{i, j} \frac{\partial u_{i}}{\partial x_{j}} d s+I_{o}$, is time integrable within the 1-dimensional stream since it represents the zero-net change in total mechanical energy per unit mass of the fluid, as (Eq.12) shown below.

$$
\begin{gathered}
\int_{0}^{t} \frac{d B(\vec{x}(s), s)}{d s} d s=B(\vec{x}(t), t)-B\left((\vec{x}(0), 0)=\left.\Delta B\right|_{0} ^{t}=0\right. \\
\left.\Delta B\right|_{0} ^{t}=\frac{1}{2} u_{i}^{2}(\vec{x}(t), t)+\phi(x(t))+\frac{p(\vec{x}(t))}{\rho_{o}}+\frac{1}{\rho_{o}} \int_{0}^{t} \tau_{i, j} \frac{\partial u_{i}}{\partial x_{j}} d s+I_{o}-\frac{1}{2} u_{i}^{2}\left(\vec{x}_{o}, 0\right)-\phi\left(\vec{x}_{o}\right)-\frac{p\left(\vec{x}_{o}\right)}{\rho_{o}}- \\
I_{o}=0
\end{gathered}
$$

The Bernoulli formula results for Eulerian coordinates could also have been simply obtained by multiplying each Navier Stokes component equation with the corresponding fluid component, adding the three equations, integrating in time, and then performing a spatial integration with a constant finite stream volume for a very small time, $\delta t$, so the Jacobian, $\frac{\partial\left(X_{1}, X_{2}, X_{3}\right)}{\partial\left(R_{1}, R_{2}, R_{3}\right)}$, is a constant $\sim 1$ since the control volume is nearly static and therefore integrating by parts the shear stress with no slip condition or shear stress of zero at the boundary, realizing the terms are perfect time derivative and using the fundamental theorem of calculus [5], except for the shear stress term in which the time integral remains ${ }^{4}$.

$$
\begin{gathered}
\iiint_{\delta V_{o}} \int_{0}^{\delta t}\left(\frac{\partial u_{i}}{\partial s}+u_{j} \frac{\partial u_{i}}{\partial X_{j}}+\frac{\partial \phi}{\partial X_{i}}+\frac{1}{\rho_{o}} \frac{\partial p}{\partial X_{i}}-\frac{1}{\rho_{o}} \frac{\partial \tau_{i, j}}{\partial X_{j}}\right) u_{i} d s \rho_{o} d V=\iiint_{\delta V_{0}} \int_{0}^{\delta t} \frac{D B(\vec{X}(s), s)}{D s} d s \rho_{o} d V \\
=0
\end{gathered}
$$

Therefore, the Bernoulli equation is the first time integral of the Navier Stokes equations, i.e., the law of conservation of energy per unit mass, which holds as long as the resulting surface integral is zero $\left(\iint_{S_{0}} \tau_{i, j} u_{i} n_{j} d S=0\right)$ due to nonslip condition for solid boundaries or require the fluid shear stress to be null since the surface normal may be arbitrary in absence of solid boundaries.

$$
\iiint_{V_{o}} \int_{0}^{\delta t}\left[\left(\frac{\partial u_{i}}{\partial s}+u_{j} \frac{\partial u_{i}}{\partial X_{j}}+\frac{\partial \phi}{\partial X_{i}}+\frac{1}{\rho_{o}} \frac{\partial p}{\partial X_{i}}-\frac{1}{\rho_{o}} \frac{\partial \tau_{i, j}}{\partial X_{j}}\right) u_{i}-\frac{D B(\vec{X}(s), s)}{D s}\right] d s \rho_{o} d V=0
$$

\footnotetext{
${ }^{4}$ Similar process used to obtain (Eqs. 6-7ABCDEF), see Appendix A for formal derivation.
} 


\section{Javier Rivera}

The integrand is zero ${ }^{5}$ within the stream control volume. (See Section 3.1 and Appendix A, for formal proof)

$$
\frac{D B(\vec{X}(t), t)}{D t}-\left(\frac{\partial u_{i}}{\partial t}+u_{j} \frac{\partial u_{i}}{\partial X_{j}}+\frac{\partial \phi}{\partial X_{i}}+\frac{1}{\rho_{o}} \frac{\partial p}{\partial X_{i}}-\frac{1}{\rho_{o}} \frac{\partial \tau_{i, j}}{\partial X_{j}}\right) u_{i}=0
$$

By carrying out the material or field derivative of the Bernoulli equation and considering term-by-term comparison with the results in Appendix A to obtain cancellations of equal terms. Therefore, within the 1-dimensional stream the sampled Eulerian and Lagrangian coordinates are equivalent by construction, the material derivative is conjectured to be equivalent to the field derivative within the stream developed in Section 3.2. See Section 3.2 for a formal demonstration and notation of the field derivative used below.

$$
\begin{gathered}
\int_{0}^{t} \frac{D B(\vec{X}(s), s)}{D s} d s=\int_{0}^{t} \frac{d B(s, \vec{x}(s), \vec{u}(\vec{X}(s), s))}{d s} d s \\
=B(t, \vec{x}(t), \vec{u}(\vec{X}(t), t))-B\left(0, \vec{x}(0), \vec{u}(\vec{X}(0), 0)=\left.\Delta B\right|_{0} ^{t}\right. \\
\left.\Delta B\right|_{0} ^{t}=\int_{0}^{t}\left(\frac{\partial u_{i}}{\partial s}+u_{j} \frac{\partial u_{i}}{\partial X_{j}}+\frac{\partial \phi}{\partial X_{i}}+\frac{1}{\rho_{o}} \frac{\partial p}{\partial X_{i}}-\frac{1}{\rho_{o}} \frac{\partial \tau_{i, j}}{\partial X_{j}}\right) u_{i} d s=0
\end{gathered}
$$

From the examples in Section 2.2, the field derivative operator does not behave like a Lie derivative or covariant derivative (see reference [9]), thus the field derivative is not a parallel vector transport differentiation process, but rather a directional derivative which maps to the tangent space of a field function (i.e., $F(t, \vec{X}(t), \vec{u}(\vec{X}, t))$ ) in the phase-spacetime domain (7 dimensions).

Although for the Lagrangian coordinates, the time in the Bernoulli integral upper limit is assumed to be for short times, let time $t \rightarrow \infty$, in (Eq. 12), a viscous fluid without external potentials $(\phi=0)$; thus, the change in kinetic energy per unit mass will go to a small constant, the change of internal energy per unit mass will be a small constant since the fluid flow is due to thermal energy, and so will the change in potential energy per unit mass.

$$
\frac{1}{2} u_{i}^{2}(\vec{x}(\infty), \infty)-\frac{1}{2} u_{i}^{2}\left(\vec{x}_{o}, 0\right)+\frac{p(\vec{x}(\infty))-p\left(\vec{x}_{o}\right)}{\rho_{o}}+\frac{1}{\rho_{o}} \int_{0}^{\infty} \tau_{i, j} \frac{\partial u_{i}}{\partial x_{j}} d s=\Delta K+\Delta V+\Delta I=0
$$

By using the triangle inequality, the equation below indicates the absolute value of the change in internal energy density remains finite within the fluid finite volume as long as the absolute value of the change in kinetic energy density and absolute value of the change in the potential energy density are both bounded by a constant, $B$.

$$
\left|\frac{1}{\rho_{o}} \int_{0}^{\infty} \tau_{i, j} \frac{\partial u_{i}}{\partial x_{j}} d s\right| \leq \frac{1}{2}\left|u_{i}^{2}\left(\vec{x}_{o}, 0\right)-u_{i}^{2}(\vec{x}(\infty), \infty)\right|+\frac{\left|p\left(\vec{x}_{o}\right)-p(\vec{x}(\infty))\right|}{\rho_{o}} \leq|\Delta K|+|\Delta V|<B
$$

In equations 5, 6 and 7ABCDEF, the Eulerian cylindrical control volumes were used. When considering the Bernoulli formulas, the Eulerian coordinates were used for small volumes with the re-application of $(E q .7 F)$ and a sampling scheme. By construction,

\footnotetext{
${ }^{5}$ A more formal method of proof has been used, see Appendix A for formal derivation.
} 
On the Field Derivatives and its Application to Fluids

start of the Lagrangian coordinates at the Eulerian stream control volume cylindrical face was used as the limit $\delta V_{k} \rightarrow 0$ as $\delta r \rightarrow 0$, and $\delta L \rightarrow 0$ by reapplying to the $(E q .7 F)$ Eulerian stream control volume tended to zero since the stream control volumes became fluid parcels or particles $\left(\delta V_{k} \rightarrow 0\right.$, as $\delta r \rightarrow 0$ ) within a 1-dimensional stream.

Section 4.0 develops the Hamilton Jacobi partial differential equation of incompressible fluids based on a theorem in reference [11] and the calculus of variation method developed in reference [1].

Acknowledgements. The author would like to thank the anonymous reviewers and chief editor of APAM for their time and effort spent in reviewing this article.

\section{Appendix A: Material derivative of the total mechanical energy}

Taking the material derivative (using the symbol provided by Batchelor [7]) of the total mechanical energy,

$$
\frac{D}{D t} E_{T}(t)=\iiint_{\delta V_{o}} \frac{D}{D t}(B(\vec{X}(t), t)) \rho_{o} \frac{\partial\left(X_{1}, X_{2}, X_{3}\right)}{\partial\left(R_{1}, R_{2}, R_{3}\right)} d V_{o}=\iiint_{\delta V_{o}} \frac{D}{D t}(B(\vec{X}(t), t)) \rho_{o} d V=0
$$

Since the control volume is a finite static constant the material derivative and the integral commute. Notice the Jacobian for incompressible fluids may be a constant near unity. Using definition of Material Derivative Equation \#48 from reference [6] in page 20 (here the Batchelor symbol for material derivative operator is being used instead of reference [6] suggested operator symbol, $\frac{\delta}{\delta t}$, since this symbol can be confused with the variational derivative operator in reference [1].

$$
\frac{D}{D t}(B(\vec{X}(t), t)) \equiv \lim _{\Delta t \rightarrow 0} \frac{B(\vec{X}, t+\Delta t)-B(\vec{X}, t)}{\Delta t}+\lim _{\Delta t \rightarrow 0} \vec{u} \cdot \nabla_{\vec{X}} B(\vec{X}, t+\Delta t)
$$

Taking the first limit, the total time derivative,

$$
\lim _{\Delta t \rightarrow 0} \frac{B(\vec{X}, t+\Delta t)-B(\vec{X}, t)}{\Delta t}=\left.\frac{d B(t, \vec{X})}{d t}\right|_{\text {fixed } \vec{X}}=\frac{\partial \mathrm{B}}{\partial \mathrm{t}}=u_{i} \frac{\partial u_{i}}{\partial \mathrm{t}}+\frac{1}{\rho_{o}} \tau_{i, j} \frac{\partial u_{i}}{\partial X_{j}}
$$

Since $\frac{\partial}{\partial t}\left\{\frac{1}{2} u_{i}^{2}(\vec{X}, t)\right\}=u_{i} \frac{\partial u_{i}}{\partial t}$ by chain rule of differentiation or by definition below.

By Taylor series expansion

$$
\frac{\partial}{\partial t}\left\{\frac{1}{2} u_{i}^{2}(\vec{X}, t)\right\}=\frac{1}{2} \lim _{\Delta t \rightarrow 0} \frac{u_{i}^{2}(\vec{X}, t+\Delta t)-u_{i}^{2}(\vec{X}, t)}{\Delta t}
$$

$$
\begin{gathered}
u_{i}(\vec{X}, t+\Delta t)=u_{i}(\vec{X}, t)+\frac{\partial u_{i}}{\partial \mathrm{t}} \Delta t+O\left(\Delta t^{2}\right) \\
u_{i}^{2}(\vec{X}, t+\Delta t)=u_{i}^{2}(\vec{X}, t)+2 u_{i}(\vec{X}, t) \frac{\partial u_{i}}{\partial t} \Delta t+O\left(\Delta t^{2}\right)
\end{gathered}
$$

Thus,

$$
\begin{aligned}
\frac{\partial}{\partial t}\left\{\frac{1}{2} u_{i}^{2}(\vec{X}, t)\right\}= & \frac{1}{2} \lim _{\Delta t \rightarrow 0} \frac{u_{i}^{2}(\vec{X}, t+\Delta t)-u_{i}^{2}(\vec{X}, t)}{\Delta t} \\
& =\frac{1}{2} \lim _{\Delta t \rightarrow 0}\left(2 u_{i}(\vec{X}, t) \frac{\partial u_{i}}{\partial \mathrm{t}}+O(\Delta t)\right)=u_{i} \frac{\partial u_{i}}{\partial \mathrm{t}}
\end{aligned}
$$




\section{Javier Rivera}

The second limit is given by

$$
\begin{gathered}
\lim _{\Delta t \rightarrow 0} \vec{u} \cdot \nabla_{\vec{X}} B(\vec{X}, t+\Delta t)=\left.u_{j} \frac{\partial}{\partial X_{j}} B(\vec{X}, t)\right|_{\text {fixed } t}=u_{i}\left(u_{j} \frac{\partial u_{i}}{\partial X_{j}}\right)+u_{j} \frac{\partial}{\partial X_{j}} \phi(\vec{X})+u_{j} \frac{\partial}{\partial X_{j}} \frac{p(\vec{X})}{\rho_{o}} \\
\text { Since } u_{j} \frac{\partial}{\partial X_{j}}\left(\frac{1}{2} u_{i}^{2}(\vec{X}, t)\right)=u_{i}\left(u_{j} \frac{\partial u_{i}}{\partial X_{j}}\right) \text { by elementary chain rule of differentiation }
\end{gathered}
$$

Since $\mathrm{j}$ is a summation index dummy, replace the last two terms summation index to $\mathrm{i}$,

Thus,

$$
\lim _{\Delta t \rightarrow 0} \vec{u} \cdot \nabla_{\vec{X}} B(\vec{X}, t+\Delta t)=u_{j} \frac{\partial}{\partial X_{j}} B(\vec{X}, t)=u_{i}\left(u_{j} \frac{\partial u_{i}}{\partial X_{j}}\right)+u_{i} \frac{\partial}{\partial X_{i}} \phi(\vec{X})+u_{i} \frac{\partial}{\partial X_{i}} \frac{p(\vec{X})}{\rho_{o}}
$$

$$
\lim _{\Delta t \rightarrow 0} \vec{u} \cdot \nabla_{\vec{X}} B(\vec{X}, t+\Delta t)=u_{j} \frac{\partial}{\partial X_{j}} B(\vec{X}, t)=u_{i}\left(u_{j} \frac{\partial u_{i}}{\partial X_{j}}+\frac{\partial}{\partial X_{i}} \phi(\vec{X})+\frac{1}{\rho_{o}} \frac{\partial}{\partial X_{i}} p(\vec{X})\right)
$$

Adding the results of the two limits,

$$
\frac{D}{D t}(B(\vec{X}(t), t))=u_{i} \frac{\partial u_{i}}{\partial \mathrm{t}}+\frac{1}{\rho_{o}} \tau_{i, j} \frac{\partial u_{i}}{\partial X_{j}}+u_{i}\left(u_{j} \frac{\partial u_{i}}{\partial X_{j}}+\frac{\partial}{\partial X_{i}} \phi(\vec{X})+\frac{1}{\rho_{o}} \frac{\partial}{\partial X_{i}} p(\vec{X})\right)
$$

Rearranging terms,

$$
\frac{D}{D t}(B(\vec{X}(t), t))=u_{i} \frac{\partial u_{i}}{\partial \mathrm{t}}+u_{i}\left(u_{j} \frac{\partial u_{i}}{\partial X_{j}}+\frac{\partial}{\partial X_{i}} \phi(\vec{X})+\frac{1}{\rho_{o}} \frac{\partial}{\partial X_{i}} p(\vec{X})\right)+\frac{1}{\rho_{o}} \tau_{i, j} \frac{\partial u_{i}}{\partial X_{j}}
$$

Since for small time intervals, $\Delta t$, the Jacobian is nearly constant, $\frac{\partial\left(X_{1}, X_{2}, X_{3}\right)}{\partial\left(R_{1}, R_{2}, R_{3}\right)} \sim 1$, since the cylindrical control volume is nearly fixed or depends on higher order terms of $O\left(\Delta t^{2}\right)$, thus the volume, $V$, and the Jacobian can be treated as a constant since the fluid is incompressible. By integration by parts of the shear stress term, assuming the required surface boundary condition, where either the fluid velocity has a no-slip condition or the surface normal is arbitrary; therefore, the shear stress is zero at the volume surface, as expected.

$$
\iiint_{\delta V_{o}} \frac{1}{\rho_{o}} \tau_{i, j} \frac{\partial u_{i}}{\partial X_{j}} \rho_{o} d V=\oiint_{\delta S_{o}} \tau_{i, j} u_{i} n_{j} \rho_{o} d S-\iiint_{\delta V_{o}} \frac{1}{\rho_{o}} \frac{\partial \tau_{i, j}}{\partial X_{j}} u_{i} \rho_{o} d V=-\iiint_{\delta V_{o}} \frac{1}{\rho_{o}} \frac{\partial \tau_{i, j}}{\partial X_{j}} u_{i} \rho_{o} d V
$$

Thus, the material derivative result of the total mechanical energy results in the Navier Stokes equations.

$$
\begin{gathered}
\frac{D}{D t} E_{T}(t)=\iiint_{\delta V_{o}} \frac{D}{D t}(B(\vec{X}(t), t)) \rho_{o} d V=\iiint_{\delta V_{o}} u_{i}\left(\frac{\partial u_{i}}{\partial t}+u_{j} \frac{\partial u_{i}}{\partial X_{j}}+\frac{\partial \phi}{\partial X_{i}}+\frac{1}{\rho_{o}} \frac{\partial p}{\partial X_{i}}-\frac{1}{\rho_{o}} \frac{\partial \tau_{i, j}}{\partial X_{j}}\right) \rho_{o} d V \\
=0
\end{gathered}
$$

Taking the limit of the cylindrical control volume, $\delta V_{o}=\delta L \pi \delta r^{2}$, to zero to enclose the 1dimensional stream for a small distance, $\delta L$,

$$
\lim _{\delta V_{o} \rightarrow 0} \frac{1}{\delta V_{o}} \iiint_{\delta V_{o}} \frac{D B(\vec{X}(t), t)}{D t}-\left(\frac{\partial u_{i}}{\partial t}+u_{j} \frac{\partial u_{i}}{\partial X_{j}}+\frac{\partial \phi}{\partial X_{i}}+\frac{1}{\rho_{o}} \frac{\partial p}{\partial X_{i}}-\frac{1}{\rho_{o}} \frac{\partial \tau_{i, j}}{\partial X_{j}}\right) u_{i} \rho_{o} d V=0
$$

Yields, the desired result by reference [2] footnote on page 71 (i.e. use of Mean Value Theorem for Integrals) valid within every point in the 1-dimensional stream.

$$
\frac{D B(\vec{X}(t), t)}{D t}-\left(\frac{\partial u_{i}}{\partial t}+u_{j} \frac{\partial u_{i}}{\partial X_{j}}+\frac{\partial \phi}{\partial X_{i}}+\frac{1}{\rho_{o}} \frac{\partial p}{\partial X_{i}}-\frac{1}{\rho_{o}} \frac{\partial \tau_{i, j}}{\partial X_{j}}\right) u_{i}=0
$$


On the Field Derivatives and its Application to Fluids

\section{REFERENCES}

1. J.Rivera, A variational principle for incompressible viscous fluids, Developments in Theoretical and Applied Mechanics (2002), River Cross Publishing, Orlando, FL p. 479-488.

2. L.M.Milne-Thomson, Theoretical Hydrodynamics, Fourth Edition, The Macmillan Company, New York, 1960, Footnote on Page 71.

3. H.Jänicke and G.Scheuermann, Measuring Complexity in Lagrangian and Eulerian Flow Descriptions, Computer Graphics forum, Vol. 0, 1981, No. 0, page 3.

4. G. E.Andrews, Number Theory, Dover Publications, New York, 1971, page 3

5. W.F.Trench, Advance Calculus, Harper \& Row, NY, NY, 1978

6. E.A.Ivanova, E.N.Vilchevskaya and W.H.Müller, Time Derivatives in Material and Spatial Description - What Are the Differences and Why Do They Concern Us?, Advance Methods of Continuum Mechanics for Materials and Structures, Advance Structured Materials Volume 60, Springer Science + Business Media, Singapore, 2016, pp. 3-28

7. G.K.Batchelor, An Introduction to Fluid Dynamics, Cambridge Mathematical Library, Cambridge University Press, Cambridge, UK, 1999, page 72

8. T. Levi-Civita, The Absolute Calculus, Dover Publications, New York, pp. 13-60

9. R.d'Inverno, Introducing Einstein Relativity, Clarendon Press, Oxford, UK, 1992, pp. 69-89

10. J.Serrin, Mathematical Principles of Classical Fluid Mechanics, Handbuk der Physik, VIII, Spring-Verlag, 1959, pp. 131

11. I.M.Gelfand and S.V.Fomin, Calculus of Variations, Prentice-Hall, Inc., Engelwood Cliffs, N.J., 1963, pp. 131-136

12. D.D.Holm, I am very grateful to Professor Holm for his time and personal comments of an earlier version of [1] in 1999. 